\title{
BGK Polyatomic Model for Rarefied Flows
}

\author{
Florian BERNARD ${ }^{1}$, Angelo IOLLO ${ }^{1}$, and Gabriella PUPPO ${ }^{2}$ \\ ${ }^{1}$ Univ. Bordeaux, IMB, UMR 5251, F-33400 Talence, France., INRIA, Team MEMPHIS, F-33400 \\ Talence, France. \\ ${ }^{2}$ Università dell'Insubria, Como
}

\begin{abstract}
In this work we present a new model of BGK type for polyatomic gases. The model incorporates the different relaxation rates of translational, rotational and/or vibrational modes characterizing polyatomic molecules using a BGK-type equation, and additional relaxation equations for the temperatures associated to each internal energy mode. We construct an efficient numerical scheme which is implicit in the relaxation terms, and test the model and the scheme on several problems, confirming the Asymptotic Preserving properties of the scheme, and comparing the results provided by the model with experimental and DSMC simulations, carried out on the full Boltzmann polyatomic equation.
\end{abstract}

Keywords: Kinetic models, Polyatomic gas, Multi-temperature, BGK model, AP schemes

\section{Introduction}

We propose a simple model based on the BGK equation, extended to include polyatomic gases. The energy of a monoatomic gas, in the absence of an external field, is stored entirely in the kinetic energy of its molecules. In a polyatomic gas, energy is stored not only in the molecules speed, but also in their internal rotational and vibrational modes. The state of a monoatomic molecule can be completely described by the trajectory of a point in a phase space composed of the different space directions available, and the corresponding directions of motion. The passage from monoatomic to polyatomic gases implies that additional degrees of freedom should be considered, because now the state of each molecule is given not only by its position and speed, but also by its rotational and vibrational modes. Thus, the number of dimensions for the state space of a polyatomic molecule increases with the complexity of the molecule.

The evolution of a polyatomic gas in non equilibrium conditions is clearly extremely important in applications. In many cases, for instance, air can be modelled as a gas composed of biatomic molecules. However, most kinetic models deal with the case of a monoatomic gas. The extension to the polyatomic case has been studied especially in the context of the full Boltzmann equation, see for example the classic text by Cercignani, [13].

The structure of the Boltzmann equation is extremely complex, and its complexity naturally increases for polyatomic gases. Thus, there is a strong interest to develop simplified models for non equilibrium flows of polyatomic molecules, especially with the purpose of developing effective and fast numerical methods, to accurately compute solutions of interest for engineering applications.

A simplified kinetic model which is particularly effective in scientific computing is the BGK model, [9]. BGK is derived from Boltzmann equation, assuming that the relaxation to equilibrium is very fast, or that the flow is close to equilibrium. However, it is known that BGK 
provides good approximations to rarefied flows even in non equilibrium conditions, see the recent [17], or [26, 2]. The more refined ES-BGK model moreover provides correct estimates for the Prandtl number, and, as standard BGK, can be proven to satisfy entropy decay, [4]. The popularity of BGK-like models is due to the fact that they provide the correct asymptotic limits, but can also model effectively moderately non-equilibrium regimes.

The standard BGK model applies to a monoatomic gas. In the polyatomic case, it is necessary to take into account the internal degrees of freedom of polyatomic molecules. The collisions between molecules cannot be considered as elastic, because they result not only in kinetic energy exchanges between the molecules, but they also involve exchanges between rotational and vibrational energies. Polyatomic corrections to kinetic models can be found in [10] or [14]. Here we are more interested in the extension of BGK models to include polyatomic effects, see [27], or [11] for an extension to the polyatomic case of the ES-BGK model. A possible alternative is [23], which is used in [26] and [17], which, however, does not guarantee the positivity of the distribution function.

Unlike previous models, we consider a BGK operator which relaxes on a local Maxwellian, depending on different temperatures. This is coupled with a scalar relaxation equation that drives the temperatures associated to the different degrees of freedom towards a global temperature. The scalar equation is obtained imposing total energy conservation. We prove an $\mathrm{H}$-theorem for this model, which guarantees that the model satisfies the second principle of thermodynamics, and that all temperatures remain positive thouroughout the evolution of the gas $(\S 2)$. We simplify the model considering an aggregate description of the dependence on the internal energy degrees of freedom, based on reduced distribution functions in the spirit of [15]. Next, in $\S 3$, we derive an Asymptotic Preserving scheme, in the sense of [16]. In this fashion, the numerical solution will capture the correct asymptotic. The scheme is based on [21], and is implicit in the relaxation terms. The properties of the scheme and of the model are studied in $\S 3$, where we study the solution of typical shock tube problems, for different $\gamma$-law gases, close to the hydrodynamics regime. Then, we consider the shock structure, showing the effect of the different collision frequencies characterizing the internal and the translational modes. We close with two-dimensional problems, derived from aerospace applications.

The collision operator of the BGK model describes a relaxation towards a local Maxwellian, determined by the density, the macroscopic local speed and temperature. To take into account polyatomic effects, we include a multiple step relaxation, assuming that the different energy degrees of freedom relax with different speeds. This means that we assume that the expected kinetic energies of the different degrees of freedom are represented by different temperatures, each relaxing with its own characteristic rate towards a final equilibrium temperature $T$. This approach has already been used by [27], but the model described here has a simpler structure. We note that the evolution of different temperatures appears also in experimental data, [24], and DSMC simulations [26].

\section{Kinetic models for polyatomic gases}

The model we study is based on the BGK approach. The standard BGK model for a monoatomic gas can be written as

$$
\frac{\partial f}{\partial t}+\boldsymbol{\xi} \cdot \nabla_{\mathbf{x}} f=\frac{1}{\tau}\left(M_{f}-f\right)
$$

where $M_{f}$ is the equilibrium distribution function in dimensionless form computed from macroscopic quantities. In the monoatomic case, it is expressed as

$$
M_{f}(\mathbf{x}, \boldsymbol{\xi}, t)=\frac{\rho(\mathbf{x}, t)}{(2 \pi T(\mathbf{x}, t))^{3 / 2}} \exp \left(-\frac{|\boldsymbol{\xi}-\mathbf{U}(\mathbf{x}, t)|^{2}}{2 T(\mathbf{x}, t)}\right) .
$$


The macroscopic quantity $\tau$ is the relaxation time that depends on local and global variables, namely the local density and temperature, but also the reference viscosity $\mu_{0}$ at the reference temperature $T_{0}$, the reference density $\rho_{0}$, the specific gas constant $R$ and the characteristic length of the problem $L$ :

$$
\frac{1}{\tau}=\frac{\sqrt{R T_{0}} \rho_{0} L}{\mu_{0}} \rho T^{1-\delta}=\frac{1}{K n_{\infty}} \rho T^{1-\delta},
$$

where $\delta$ is the exponent of the viscosity law of the gas, and $K n_{\infty}$ is the Knudsen number in reference conditions.

The macroscopic quantities (the density $\rho$, the velocity $\mathbf{U}$ and the total energy $E$ ) characterizing the flow can be recovered from the moments of $f$ :

$$
\left\{\begin{array}{l}
\rho=\int_{\mathbb{R}^{3}} f d \boldsymbol{\xi} \\
\rho \mathbf{U}=\int_{\mathbb{R}^{3}} \boldsymbol{\xi} f d \boldsymbol{\xi} \\
E=\int_{\mathbb{R}^{3}} \frac{|\boldsymbol{\xi}|^{2}}{2} f d \boldsymbol{\xi}
\end{array}\right.
$$

In the following section, we describe the extension we propose to the polyatomic case.

\subsection{A BGK based model}

The idea is to add additional energy degrees of freedom in the expression of the Maxwellian distribution function. This is done increasing the dimensions $d$ of the space of microscopic speeds. We treat these additional variables as microscopic velocities, so that their expected value at the macroscopic scale is zero, implying that they do not contribute to the macroscopic velocity of the gas. However, we can associate an internal energy to the effect of these variables on the distribution function, computing their second moment. Moreover, we consider a general case where the energy is not equally distributed between the energy degrees of freedom.

First, we state the notation that will be used throughout. Let $n$ be the number of translational degrees of freedom (which usually coincides with the number of space dimensions) and $r$ be the number of the rotational and vibrational degrees of freedom, mimicking the internal structure of the molecule, with $d=n+r$. Denote by $\boldsymbol{\eta} \in \mathbb{R}^{d}$ the vector of all energy degrees of freedom, and of these $\boldsymbol{\xi} \in \mathbb{R}^{n}$ will be used to single out the translational degrees of freedom (then $\boldsymbol{\eta}=\boldsymbol{\xi}$ for the BGK model of a monoatomic gas). Further, $\overline{\boldsymbol{\eta}} \in \mathbb{R}^{d}$ will be the macroscopic value on which the equilibrium function is centred (the macroscopic velocity $\mathbf{U}$ in the case of the monoatomic BGK model), $\boldsymbol{\lambda} \in \mathbb{R}^{d}$ is the vector of the coefficients giving the distribution of the energy between the degrees of freedom (this coincides with $1 / 2 T$ in the case of the monoatomic BGK model for each of the three translational degrees of freedom).

In this model, hence, we propose to explicitly model the dynamics of the additional rotational, vibrational and other degrees of freedom. In other approaches, like in [3], these degrees of freedom are all lumped in the internal energy. In particular, compared to [3], the main difference here is in the mechanism of relaxation of the internal and translational temperature to the equilibrium temperature. In our approach, the relaxation is explicitly ensured at macroscopic level in the source term of the transport equation for the rotational temperature. In [3], thanks to the consistency of the stress tensor with the third order moment of the Gaussian equilibrium function, the relaxation of the internal temperature to the equilibrium temperature is implicitly enforced. This would not be feasible in the present BGK model. However, the main advantage 
of our model with respect to [3] is that it is much cheaper since BGK type models are less costly than ES-BGK type models.

The polyatomic BGK model we propose reads:

$$
\begin{aligned}
& \frac{\partial f}{\partial t}+\boldsymbol{\xi} \cdot \nabla_{\mathbf{x}} f=\frac{1}{\tau}\left(M_{f}-f\right) \\
& M_{f}(\mathbf{x}, \boldsymbol{\eta}, t)=\rho(\mathbf{x}, t) \prod_{k=1, d}\left(\frac{\lambda_{k}}{\pi}\right)^{1 / 2} \exp \left(-\left(\lambda_{k}\left(\eta_{k}-\bar{\eta}_{k}\right)^{2}\right) .\right.
\end{aligned}
$$

For simplicity, we state the model for the particular case of bi-atomic molecules, which can be easily extended to more complex polyatomic gases. Here we will have three translational and two rotational degrees of freedom. Considering the same temperature for similar degrees of freedom in the case of a diatomic gas, we have:

$$
\boldsymbol{\lambda}=\left(\frac{1}{2 \Lambda}, \frac{1}{2 \Lambda}, \frac{1}{2 \Lambda}, \frac{1}{2 \Theta}, \frac{1}{2 \Theta}\right)^{T} .
$$

The evolution of $\boldsymbol{\lambda}$ is governed by the equation of energy conservation. This closure equation is obtained by the relaxation of the local Maxwellian $M_{f}$ to the equilibrium Maxwellian,

$$
\begin{aligned}
& \frac{\partial M_{f}}{\partial t}+\boldsymbol{\xi} \cdot \nabla_{\mathbf{x}} M_{f}=\frac{1}{Z_{r} \tau}\left(M_{e q}-M_{f}\right) \\
& M_{e q}(\mathbf{x}, \boldsymbol{\eta}, t)=\frac{\rho(\mathbf{x}, t)}{\left(2 \pi T_{e q}(\mathbf{x}, t)\right)^{d / 2}} \exp \left(-\frac{|\eta-\overline{\boldsymbol{\eta}}|^{2}}{2 T_{e q}(\mathbf{x}, t)}\right)
\end{aligned}
$$

Here, $Z_{r}$ is a parameter that accounts for the fact that the rotational collision frequency is a priori different from the translational collision frequency, thus the relaxation time towards a common temperature $T_{e q}$ is governed by a different characteristic time $Z_{r} \tau$ with respect to the relaxation time $\tau$ appearing in the evolution of $f$. We choose for $Z_{r}$, the model given in [18] :

$$
Z_{r}=\frac{Z_{r}^{*}}{1+\left(\pi^{3 / 2} / 2\right) \sqrt{\tilde{T} / T t}+\left(\pi+\pi^{2} / 4\right)(\tilde{T} / T t)}
$$

where the constants $Z_{r}^{*}$ and $\tilde{T}$ depends on the gas. For $N_{2}$ over a temperature range from $30 \mathrm{~K}$ to $3000 \mathrm{~K}, Z_{r}^{*}=23$ and $\tilde{T}=91.5 \mathrm{~K}$.

Multiplying the kinetic equation (6) by $\prod_{k=n+1, d} \eta_{k}$ and integrating in phase space, we obtain the evolution equation for the rotational energy,

$$
\frac{\partial}{\partial t}(\rho \Theta)+\frac{\partial}{\partial x}(\rho \mathbf{U} \Theta)=\frac{\rho}{Z_{r} \tau}\left(T_{e q}-\Theta\right),
$$

which can be simplified using mass conservation to give:

$$
\partial_{t} \Theta+\mathbf{U} \cdot \nabla \Theta=\frac{1}{Z_{r} \tau}\left(T_{e q}-\Theta\right) .
$$

The system is then closed imposing that total energy is conserved in (4) and (6). From this, the internal energy is obtained:

$$
\begin{aligned}
\rho e_{i n t} & =\int_{\mathbb{R}^{r}} \frac{1}{2} \sum_{k=1}^{d}\left(\eta_{k}-\bar{\eta}_{k}\right)^{2} f \\
& =\frac{1}{2} \rho \sum_{k=1}^{d} \frac{1}{2 \lambda_{k}} \\
& =\frac{1}{2} \rho(n \Lambda+m \Theta)=\frac{d}{2} \rho T_{e q}
\end{aligned}
$$


where we recall that $n$ and $m$ are respectively the number of translational and rotational degrees of freedom and $n+m=d$.

The full model in the case of a diatomic gas reads then:

$$
\left\{\begin{array}{l}
\frac{\partial f}{\partial t}+\boldsymbol{\xi} \cdot \nabla_{\mathbf{x}} f=\frac{1}{\tau}\left(M_{f}-f\right) \\
\partial_{t} \Theta+\mathbf{U} \cdot \nabla \Theta=\frac{1}{Z_{r} \tau}\left(T_{e q}-\Theta\right)
\end{array}\right.
$$

where $M_{f}(\mathbf{x}, \boldsymbol{\xi}, \boldsymbol{\eta}, t)=\frac{\rho}{(2 \pi \Lambda)^{n / 2}(2 \pi \Theta)^{m / 2}} \exp \left(-\frac{|\boldsymbol{\xi}-\mathbf{U}|^{2}}{2 \Lambda}-\frac{|\hat{\boldsymbol{\eta}}|^{2}}{2 \Theta}\right)$ where $\hat{\boldsymbol{\eta}}$ contains only the rotational energy degrees of freedom which have mean value zero.

Compared to [18], the proposed model has a lower computational cost since we introduce only one equilibrium distribution function which represents a very costly part in a numerical code.

\section{Properties of the polyatomic model}

In the following, we derive several important properties for the polyatomic BGK model introduced in the previous section.

\subsection{Moments}

In this model, density, momentum and total energy are conserved

$$
\begin{aligned}
& \rho=\int_{\mathbb{R}^{d}} f d \boldsymbol{\eta}=\int_{\mathbb{R}^{d}} M_{f} d \boldsymbol{\eta} \\
& \rho \overline{\boldsymbol{\eta}}=\int_{\mathbb{R}^{d}} \boldsymbol{\eta} f d \boldsymbol{\eta}=\int_{\mathbb{R}^{d}} \boldsymbol{\eta} M_{f} d \boldsymbol{\eta} \\
& E=\int_{\mathbb{R}^{d}} \frac{|\boldsymbol{\eta}|^{2}}{2} f d \boldsymbol{\eta}=\int_{\mathbb{R}^{d}} \frac{|\boldsymbol{\eta}|^{2}}{2} M_{f} d \boldsymbol{\eta} .
\end{aligned}
$$

However, the partial moments are not conserved. Considering the same ordering for the microscopic velocities we used above, we find

$$
\begin{aligned}
& \frac{n}{2} \rho T_{t}=\int_{\mathbb{R}^{d}} \frac{1}{2} \sum_{k=1}^{n}\left(\eta_{k}-\bar{\eta}_{k}\right)^{2} f d \boldsymbol{\eta} \quad \text { and } \quad \frac{n}{2} \rho \Lambda=\int_{\mathbb{R}^{d}} \frac{1}{2} \sum_{k=1}^{n}\left(\eta_{k}-\bar{\eta}_{k}\right)^{2} M_{f} d \boldsymbol{\eta} \\
& \frac{m}{2} \rho T_{r}=\int_{\mathbb{R}^{d}} \frac{1}{2} \sum_{k=n+1}^{d}\left(\eta_{k}-\bar{\eta}_{k}\right)^{2} f d \boldsymbol{\eta} \quad \text { and } \quad \frac{m}{2} \rho \Theta=\int_{\mathbb{R}^{d}} \frac{1}{2} \sum_{k=n+1}^{d}\left(\eta_{k}-\bar{\eta}_{k}\right)^{2} M_{f} d \boldsymbol{\eta} .
\end{aligned}
$$

Thus the model must provide the time evolution not only of the distribution function, but also of all partial temperatures. This is the task of the evolution equation for $\Theta$ in (12), which, together with the conservation of total energy, is enough to provide the time evolution of all temperatures involved in the model. 


\subsection{Positivity}

In this section, we show that the model preserves the positivity of the distribution function and of all partial temperatures in the space homogeneous case. In this case, the model reduces to

$$
\begin{aligned}
& \partial_{t} f=\frac{1}{\tau}\left(M_{f}-f\right) \\
& \partial_{t} \Theta=\frac{1}{Z_{r} \tau}\left(T_{e q}-\Theta\right)
\end{aligned}
$$

with $f_{0}$ and $\Theta_{0}$ as positive initial conditions for $f$ and $\Theta$ respectively. Since in the space homogeneneous case, the total energy is a constant, we also have $\partial_{t} T_{e q}=0$. Moreover, mass is conserved. Thus, if at the initial time, the density is positive, the Maxwellians $M_{f}$ and $M_{e q}$ remain positive.

Multiplying the first equation of the homogeneous model (18) by $e^{t / \tau}$, we get

$$
\partial_{t}\left(e^{t / \tau} f\right)=\frac{1}{\tau} M_{f} e^{t / \tau}
$$

which, integrating between 0 and $t$, yields

$$
f(\boldsymbol{\xi}, \boldsymbol{\eta}, t)=f_{0}(\boldsymbol{\xi}, \boldsymbol{\eta}) e^{-t / \tau}+\frac{1}{\tau} \int_{0}^{t} e^{s / \tau} M_{f}(\boldsymbol{\xi}, \boldsymbol{\eta}, s) d s .
$$

Provided the initial condition satisfies $f_{0} \geq 0, M_{f}$ remains positive, and the previous equation implies $f \geq 0$.

We use the same technique to prove the preservation of positivity for all partial temperatures. We multiply the equation for the rotational temperature (19) by $e^{t /\left(Z_{r} \tau\right)}$. Since the equilibrium temperature $T_{e q}$ is constant,

$$
\Theta(t)=\Theta_{0} e^{-t /\left(Z_{r} \tau\right)}+T_{e q}\left(1-e^{-t /\left(Z_{r} \tau\right)}\right),
$$

which proves that the rotational equilibrium temperature remains positive, provided $\Theta_{0} \geq 0$. A similar argument shows the positivity of $\Lambda$, again if $\Lambda \geq 0$ at the initial time.

\subsection{The $\mathcal{H}$ theorem}

One of the most important properties of the Boltzmann equation is that it respects the $\mathcal{H}$ theorem, which governs the evolution towards equilibrium of the solution. It means that the second principle of the thermodynamics is satisfied. To be valid, our model should also respect this theorem.

Let us define the $\mathcal{H}$-function as:

$$
\mathcal{H}(f)=\int_{\mathbb{R}^{d}} f \log f d \boldsymbol{\eta}+Z_{r} \int_{\mathbb{R}^{d}} M_{f} \log M_{f} d \boldsymbol{\eta}
$$

H-theorem. Let $f$ be the solution of the polyatomic model (1). Consider an isolated gas enclosed in a domain $\Omega_{x}$ such that:

$$
\int_{\partial \Omega_{x}} \int_{\mathbb{R}^{d}}\left(\boldsymbol{\xi}-\boldsymbol{u}_{b}\right) \cdot \boldsymbol{e} f \log f d \boldsymbol{\eta} d S=0
$$

where $\boldsymbol{u}_{b}$ is the velocity of the boundaries of $\Omega$ and $\boldsymbol{e}$ is the outward pointing normal to $\partial \Omega_{\boldsymbol{x}}$. Assume that $Z_{r}>0$, and that the initial temperatures are well prepared, in the sense that $T_{r}(0)=\Theta(0)$. Suppose further that, at the initial time, $f(., ., t=0) \geq 0$, then

$$
\frac{d H}{d t} \leqslant 0
$$


for all time, where $H[f]=\int_{\Omega_{x}} \mathcal{H} d \boldsymbol{x}$. Moreover $\frac{d H}{d t}=0$, if and only if $f=M_{e q}$.

Proof. We prove the theorem for the space homogeneous case. The general case then follows from the proof of the $\mathrm{H}$-theorem for the standard BGK model, [3]. Since density, momentum and total energy are conserved, the equation for the rotational energy can be rewritten as

$$
\partial_{t} M_{f}=\frac{1}{Z_{r} \tau}\left(M_{e q}-M_{f}\right) .
$$

Let us multiply equation (18) by $(1+\log f)$ and integrate over the space of all microscopic velocities $\Omega_{\boldsymbol{\eta}}$. Then multiply $(22)$ by $Z_{r}\left(1+\log M_{f}\right)$, integrate in velocity space and add the two results. We obtain:

$$
\frac{d \mathcal{H}}{d t}=\frac{1}{\tau} \int_{\mathbb{R}^{d}}\left(M_{f}-f\right)(1+\log f) d \boldsymbol{\eta}+\frac{1}{\tau} \int_{\mathbb{R}^{d}}\left(M_{e q}-M_{f}\right)\left(1+\log M_{f}\right) d \boldsymbol{\eta} .
$$

Since the first moment of $M_{f}, f$ and $M_{e q}$ coincide, the right hand side can be rewritten as

$$
\frac{d \mathcal{H}}{d t}=\frac{1}{\tau} \underbrace{\int_{\mathbb{R}^{d}}-\left(f-M_{f}\right)\left(\log f-\log M_{f}\right) d \boldsymbol{\eta}}_{A}+\frac{1}{\tau} \underbrace{\int_{\mathbb{R}^{d}}\left(M_{e q}-f\right) \log M_{f} d \boldsymbol{\eta}}_{B}
$$

The term $A$ is clearly negative, due to the convexity of the log function. We continue evaluating the sign of $B$. To this end, we subtract the quantity $\int\left(M_{e q}-f\right) \log M_{e q}$, which is zero, due to conservation of mass, momentum and total energy. The integrals can be explicitly calculated, and we find

$$
B=\int_{\mathbb{R}^{d}}\left(M_{e q}-f\right) \log \frac{M_{f}}{M_{e q}} d \boldsymbol{\eta}=\frac{1}{4} \rho \log \frac{T_{e q}^{n+m}}{\Lambda^{n} \Theta^{m}}\left[n\left(\frac{1}{T_{e q}}-\frac{1}{\Lambda}\right)\left(T_{e q}-T_{t}\right)+m\left(\frac{1}{T_{e q}}-\frac{1}{\Theta}\right)\left(T_{e q}-T_{r}\right)\right] .
$$

Conservation of total energy implies that $(n+m) T_{e q}=n T_{t}+m T_{r}$, thus $B$ can be rewritten as

$$
B=-\frac{1}{4} \rho \log \frac{T_{e q}^{n+m}}{\Lambda^{n} \Theta^{m}}\left[n \frac{1}{\Lambda}\left(T_{e q}-T_{t}\right)+m \frac{1}{\Theta}\left(T_{e q}-T_{r}\right)\right] .
$$

Conservation of total energy implies also that $T_{e q}$ can be written as a convex combination of $\Lambda$ and $\Theta$, as $T_{e q}=\frac{n}{n+m} \Lambda+\frac{m}{n+m} \Theta$. Using the convexity of the $\log$, we have $(n+m) \log T_{e q} \geq$ $n \log \Lambda+m \log \Theta$. Further, we substitute $n\left(T_{e q}-T_{t}\right)=-m\left(T_{e q}-T_{r}\right)$, obtaining

$$
\operatorname{sign}(B)=-\operatorname{sign}\left[\left(T_{e q}-T_{r}\right)\left(\frac{1}{\Theta}-\frac{1}{\Lambda}\right)\right] .
$$

Now, multiply (18) by $\boldsymbol{\eta}^{2}$ and integrate over velocity space, to obtain the evolution equation for $T_{r}$ :

$$
\partial_{t} \rho m T_{r}=\frac{1}{\tau}\left(m \rho \Theta-m \rho T_{r}\right)
$$

Eliminate the density through mass conservation, substitute the exact expression for the rotational temperature $\Theta$ from (20), and integrate the resulting linear non homogeneous ODE, finding

$$
T_{r}(t)=T_{r}(0) e^{-t / \tau}+\frac{Z_{r}}{1-Z_{r}}\left[e^{-t /\left(Z_{r} \tau\right)}-e^{-t / \tau}\right]\left(T_{e q}-\Theta_{0}\right)+T_{e q}\left(1-e^{-t / \tau}\right)
$$


Let us choose a well-prepared initial condition for the relaxation rotational temperature $\Theta_{0}$, namely $\Theta_{0}=T_{r}(0)$. Rearranging terms, we find

$$
T_{e q}-T_{r}(t)=\frac{1}{1-Z_{r}} e^{-t / \tau}\left(1-Z_{r} e^{-\frac{t}{\tau} \frac{1-Z_{r}}{Z_{r}}}\right)\left(T_{e q}-\Theta_{0}\right)
$$

If we suppose that $Z_{r} \neq 1$, one easily finds

$$
\operatorname{sign}\left(T_{e q}-T_{r}(t)\right)=\operatorname{sign}\left(T_{e q}-\Theta_{0}\right),
$$

and the same holds if $Z_{r} \rightarrow 1$. In fact, in this case the model reduces to the standard BGK model with $d$ degrees of freedom. Using again (20), we have

$$
\Lambda(t)-\Theta(t)=\frac{n+m}{n} e^{-t /\left(Z_{r} \tau\right)}\left(T_{e q}-\Theta_{0}\right) .
$$

Finally, since the temperatures are positive, substituting (24) and the previous result in the expression for $B$, we have

$$
\operatorname{sign}(B)=-\operatorname{sign}^{2}\left(T_{e q}-\Theta_{0}\right)
$$

which proves that the entropy production is negative.

Clearly, if $f=M_{f}=M_{e q}$, then $\frac{d \mathcal{H}}{d t}=0$. On the other hand, we have proved that $\frac{d \mathcal{H}}{d t}$ is composed of two terms which are the integrals of two non positive functions. The first integral, $A$ in (23) is zero if and only if $f=M_{f}$, while the second term is zero if and only if $f=M_{e q}$, in both cases due to the convexity of the log. Thus, if $\frac{d \mathcal{H}}{d t}=0$, then $f=M_{f}=M_{e q}$. This proves that at the final equilibrium $f$ is a Maxwellian, with all temperatures equal to $T_{e q}$.

Remark 1. Well prepared temperatures. If the initial temperatures are not well prepared, the expression for $T-T_{r}(t)$ becomes slightly more complicated:

$$
T_{e q}-T_{r}(t)=\left[T_{e q}-T_{r}(0)+\frac{1}{1-Z_{r}}\left(1-e^{-\frac{t}{\tau} \frac{1-Z_{r}}{Z_{r}}}\right)\left(T_{e q}-\Theta_{0}\right)\right] e^{-t / \tau} .
$$

To prove the entropy decay we need to assume that $\operatorname{sign}\left(T_{e q}-T_{r}(0)\right)=\operatorname{sign}\left(T_{e q}-\Theta_{0}\right)$. Thus, the theorem can be generalized to include this condition.

\subsection{The reduced model}

Let $D$ be the number of space dimensions, while $d$ is, as before, the total number of energy degrees of freedom, that is the distribution function $f$ depends on $D+d$ independent variables, plus time. This makes the problem extremely complex from a computational point of view, due to its high dimensionality. However, it is possible to reduce the number of dimensions of the distribution function with Chu's reduction, [15].

In the standard BGK model, Chu's reduction can be applied whenever the distribution function $f$ depends only on $r<D$ degrees of freedom in space. Then it is possible to rewrite the kinetic equation using only $r$ degrees of freedom, also in the microscopic velocity space. For example, in a two dimensional problem in space, the number of independent variables can be reduced to four plus time (two in space and two in microscopic velocity).

We review Chu's reduction, outlining how it is applied to the present case. In the case of a bi-atomic molecule, we will apply the reduction to aggregate the internal energy degrees of freedom. Let us consider the case in which we want to reduce the $m$ rotational degrees of freedom, while the system has $n$ translational degrees of freedom, with $n+m=d$. Let us 
label the indices pertaining to the translational and the rotational degrees of freedom as 1 to $n=d-m$ and $d-m+1$ to $d$, respectively. Correspondingly, the microsocopic velocities will be labelled as $\left(\xi_{1}, \ldots, \xi_{n}, \eta_{1}, \ldots, \eta_{m}\right)=(\boldsymbol{\xi}, \boldsymbol{\eta})$. We introduce two reduced distribution functions such that:

$$
\begin{aligned}
f_{1}(x, \boldsymbol{\xi}, t) & =\int_{\mathbb{R}^{m}} f(x, \boldsymbol{\xi}, \boldsymbol{\eta}, t) d \boldsymbol{\eta} \\
f_{2}(x, \boldsymbol{\xi}, t) & =\int_{\mathbb{R}^{m}} \frac{1}{2} \sum_{l=1}^{m} \eta_{l}^{2} f(x, \boldsymbol{\xi}, \boldsymbol{\eta}, t) d \boldsymbol{\eta}
\end{aligned}
$$

The model reduces to a system of two equations:

$$
\begin{aligned}
& \frac{\partial f_{1}}{\partial t}+\boldsymbol{\xi} \cdot \nabla_{\mathbf{x}} f_{1}=\frac{1}{\tau}\left(M_{f_{1}}-f_{1}\right) \\
& \frac{\partial f_{2}}{\partial t}+\boldsymbol{\xi} \cdot \nabla_{\mathbf{x}} f_{2}=\frac{1}{\tau}\left(M_{f_{2}}-f_{2}\right)
\end{aligned}
$$

where the reduced Maxwellians are expressed as:

$$
\begin{aligned}
& M_{f_{1}}(x, \boldsymbol{\xi}, t)=\int_{\mathbb{R}^{m}} M_{f}(x, \boldsymbol{\xi}, \boldsymbol{\eta}, t) d \boldsymbol{\eta} \\
& M_{f_{2}}(x, \boldsymbol{\xi}, t)=\int_{\mathbb{R}^{m}} \frac{1}{2} \sum_{l=1}^{m} \eta_{l}^{2} M_{f}(x, \boldsymbol{\xi}, \boldsymbol{\eta}, t) d \boldsymbol{\eta} .
\end{aligned}
$$

Their analytical expressions can be calculated by recalling that the value $\bar{\eta}_{k}$ on the reduced dimensions is zero:

$$
\begin{aligned}
& M_{f_{1}}=\rho \prod_{k=1}^{n}\left(\frac{\lambda_{k}}{\pi}\right)^{1 / 2} \exp \left(-\lambda_{k}\left(\eta_{k}-\bar{\eta}_{k}\right)^{2}\right)=\frac{\rho}{(2 \pi \Lambda)^{n / 2}} \exp \left(-\frac{(\boldsymbol{\xi}-\boldsymbol{U})^{2}}{2 \Lambda}\right) \\
& M_{f_{2}}=\sum_{k=d-m+1}^{d} \frac{1}{4 \lambda_{k}} M_{f_{1}}=\frac{m \Theta}{2} M_{f_{1}} .
\end{aligned}
$$

Equation (8) is also needed to close the system. The total temperature $T_{e q}$ needed in (8) is obtained from the total internal energy of the system, which is given by

$$
\frac{d}{2} \rho T_{e q}=\int_{\mathbb{R}^{n}} \frac{1}{2}(\boldsymbol{\xi}-\boldsymbol{U})^{2} f_{1} d \boldsymbol{\xi}+\int_{\mathbb{R}^{n}} f_{2} d \boldsymbol{\xi} .
$$

The coupled system (27), together with (8) and the coupling condition (31) is equivalent to (12), but the unknowns $f_{1}$ and $f_{2}$ depend only on $x, \boldsymbol{\xi}$ and $t$.

In $1 \mathrm{D}$ and $2 \mathrm{D}$ problems in space, we cannot reduce the residual translational degrees of freedom together with the rotational degrees of freedom, because otherwise, the model would not allow to recover the translational and rotational temperatures from the reduced distribution functions. Only the equilibrium temperature could be computed in that case, since the first distribution function $f_{1}$ would have a portion of the translational energy while the second reduced distribution function $f_{2}$ would contain the remaining part of the translational energy, along the reduced spatial dimensions, together with the rotational energy. It would then be impossible to extract the contribution of the different temperatures, from the variance of $f_{2}$.

For 1D and 2D problems, we can still reduce the dimensions of the translational degrees of freedom, to 1 and 2 dimensions respectively, provided we apply Chu's reduction in two steps. We introduce one more distribution function, giving the translational energy along the 
reduced dimensions. Thus, the first reduced distribution function $f_{1}$ will contain all information about density, momentum, and the translational energy corresponding to the degrees of freedom remained in the reduced model, the second distribution function $f_{2}$ will be used to account for the rest of the translational energy, while the third one $f_{3}$ will give the rotational energy. In $3 \mathrm{D}$, there is no need of the second distribution function, and then we will use only the two reduced distributions $f_{1}$ and $f_{2}$, as described previously. If $f_{1}, f_{2}, f_{3}$ are the reduced distribution functions in $1 \mathrm{D}$ or $2 \mathrm{D}$ (with $M_{1}, M_{2}, M_{3}$ their corresponding equilibrium functions). Let $r$ be the number of the degrees of freedom retained in the model, and $D$ be the number of space dimensions, then

$$
\begin{aligned}
f_{1} & =\int_{\mathbb{R}^{d-r}} f \prod_{k=r+1}^{d} d \eta_{k} \quad \text { and } & M_{1}=\rho \prod_{k=1}^{r}\left(\frac{\lambda_{k}}{\pi}\right)^{1 / 2} \exp \left(-\lambda_{k}\left(\eta_{k}-\bar{\eta}_{k}\right)^{2}\right) \\
f_{2}=\int_{\mathbb{R}^{d-r}} \frac{1}{2} \sum_{l=r+1}^{D} \eta_{l}^{2} f \prod_{k=r+1}^{d} d \eta_{k} & \text { and } & M_{2}=\sum_{k=r+1}^{D} \frac{1}{4 \lambda_{k}} M_{1} \\
f_{3}=\int_{\mathbb{R}^{d-r}} \frac{1}{2} \sum_{l=D+1}^{d} \eta_{l}^{2} f \prod_{k=r+1}^{d} d \eta_{k} & \text { and } & M_{3}=\sum_{k=D+1}^{d} \frac{1}{4 \lambda_{k}} M_{1}
\end{aligned}
$$

Again, equation (8) is also needed to close the system. The total temperature $T_{e q}$ appearing in (8) is obtained from the total internal energy of the system, which now is given by

$$
\frac{d}{2} \rho T_{e q}=\int_{\mathbb{R}^{r}} \frac{1}{2} \sum_{k=1}^{r}\left(\eta_{k}-\bar{\eta}_{k}\right)^{2} f_{1} \prod_{k=1}^{r} d \eta_{k}+\int_{\mathbb{R}^{r}} f_{2} \prod_{k=1}^{r} d \eta_{k}+\int_{\mathbb{R}^{r}} f_{3} \prod_{k=1}^{r} d \eta_{k} .
$$

For more complex molecules, this procedure can be extended quite easily. The reduced model would then consist of one more kinetic equation for each set of degrees of freedom resulting in the same partial temperature, together with one more macroscopic evolution equation for each partial temperature.

\section{Numerical methods}

We present the space and time discretization in the $1 \mathrm{D}$ case, so that we can also illustrate how the reduced system (32) is discretized in velocity space, enforcing exact conservation also at the discrete level. The extension to multidimensional cases is straightforward, since Cartesian grids are chosen to take advantage of easy parallelization.

\subsection{Velocity space discretization}

We consider a bi-atomic gas, in 1D in space. Thus $d=5$ (total number of degrees of freedom), $m=2$ (number of rotational degrees of freedom), and $r=1$, i.e. only one degree of freedom in space is retained, out of $D=3$. By construction, the reduced distribution function $f_{1}$ and the reduced Maxwellian $M_{1}$ satisfy:

$$
\int_{\mathbb{R}^{r}} M_{1}\left(\begin{array}{l}
1 \\
\boldsymbol{\xi}
\end{array}\right) d \boldsymbol{\xi}=\left(\begin{array}{l}
\rho(\mathbf{x}, t) \\
\rho(\mathbf{x}, t) \mathbf{U}(\mathbf{x}, t)
\end{array}\right)=\int_{\mathbb{R}^{r}} f\left(\begin{array}{l}
1 \\
\boldsymbol{\xi}
\end{array}\right) d \boldsymbol{\xi} .
$$

This is an essential property to ensure conservation of mass and momentum. Conservation of energy is more complicated. We recover the equilibrium temperature $T_{e q}$ from (33)

$$
\frac{d}{2} \rho T_{e q}=\int_{\mathbb{R}^{r}} \frac{1}{2}(\boldsymbol{\xi}-\mathbf{U})^{2} f_{1} d \boldsymbol{\xi}+\int_{\mathbb{R}^{r}} f_{2} d \boldsymbol{\xi}+\int_{\mathbb{R}^{r}} f_{3} d \boldsymbol{\xi} .
$$


With this information, we find the rotational temperature $\Theta$ from (8) and the translational temperature as $\Lambda=T_{e q}-\Theta$, with which we construct the Maxwellian $M_{1}=\rho /(2 \pi \Lambda)^{r / 2} \exp (-(\boldsymbol{\xi}-$ $\mathbf{U})^{2} /(2 \Lambda)$.

In the discrete case, a grid must be introduced in velocity space and integrals are evaluated by quadrature. Thus, conservation of mass, momentum and energy must be enforced at the discrete level. Let $\langle.,$.$\rangle denote the quadrature rule on \mathbb{R}^{r}$. Based on the work of Cabannes et al. [12] on entropic Maxwellian states, Mieussens proved in [19] that a discrete Maxwellian can be expressed as $\widetilde{M}_{f}=\exp (\boldsymbol{\alpha} \cdot \mathbf{m}(\boldsymbol{\xi}))$, such that:

$$
\left\langle\widetilde{M}_{f}(\boldsymbol{\xi}), \mathbf{m}(\boldsymbol{\xi})\right\rangle=\left(\begin{array}{c}
\rho \\
\rho \mathbf{U} \\
E
\end{array}\right)=\langle f(\boldsymbol{\xi}), \mathbf{m}(\boldsymbol{\xi})\rangle, \quad \text { with } \quad \mathbf{m}(\boldsymbol{\xi})=\left(\begin{array}{c}
1 \\
\boldsymbol{\xi} \\
\frac{1}{2}(\boldsymbol{\xi})^{2}
\end{array}\right)
$$

at the discrete level, where we have used the same symbol for the discrete moments of $f$, with a slight abuse of notation. The discrete Maxwellian distribution function $\widetilde{M}_{f}$ can then be computed as the solution of the non-linear algebraic system above solved with a NewtonRaphson algorithm. The details of this algorithm can be found in [8] or [6] in the case of the standard BGK model. A local grid technique to reduce the cost associated to the discretization in velocity as in [7] can also be applied.

To adapt it to our polyatomic model under its reduced form, it is enough to compute the total discrete energy as

$$
E=\left\langle\frac{1}{2}(\boldsymbol{\xi})^{2} f_{1}\right\rangle+\left\langle f_{2}\right\rangle+\left\langle f_{3}\right\rangle
$$

Then the total discrete internal energy is given by

$$
\frac{d}{2} \rho T_{e q}=E-\frac{1}{2} \rho \mathbf{U} .
$$

Next, substitute the discrete $T_{e q}$ into the temperature equation (8), to find the corresponding translational temperature $\Lambda$. Finally, we solve the non linear algebraic system

$$
\langle\exp (\boldsymbol{\alpha} \cdot \mathbf{m}(\boldsymbol{\xi}))\rangle=\left(\begin{array}{c}
\rho \\
\rho \mathbf{U} \\
m \Lambda
\end{array}\right), \quad \text { with } \quad \mathbf{m}(\boldsymbol{\xi})=\left(\begin{array}{c}
1 \\
\boldsymbol{\xi} \\
\frac{1}{2}(\boldsymbol{\xi}-\mathbf{U})^{2}
\end{array}\right),
$$

where $\rho=\left\langle f_{1}\right\rangle$ and $\rho \mathbf{U}=\left\langle\boldsymbol{\xi} f_{1}\right\rangle$, and $m$ is the number of rotational degrees of freedom.

We use a uniform velocity grid symmetric with respect to 0 and such that $f$ is negligible outside the grid. Hence, the trapezoidal quadrature rule is used, because it has spectral accuracy for smooth and periodic functions on a uniform grid. In 1D:

$$
\mathcal{G}_{v}=\left(\boldsymbol{\xi}_{j}\right)_{j=-n . . n} \quad \text { with } \quad \boldsymbol{\xi}_{j}=j \Delta \boldsymbol{\xi}
$$

For multidimensional cases, the same discretization is independently performed in all directions.

\subsection{Space discretization}

The physical space $\mathbf{x}$ is discretized with $\mathrm{N}$ cells of size $\Delta x$ such that $N \Delta x=x_{\text {out }}-x_{i n}, x_{i n}$ and $x_{\text {out }}$ being the boundaries of the domain. Let $\Omega_{i}$ be the $i$-th space cell. The kinetic equation is solved with a finite volume scheme, while the equation on the rotational temperature is solved with a finite difference method.

$$
\frac{\partial f_{i, j}}{\partial t}(t)+\boldsymbol{\xi}_{j} \cdot \int_{\partial \Omega_{i}} f\left(x, \boldsymbol{\xi}_{j}, t\right) \mathbf{n}_{\partial \Omega_{i}} d \sigma=\frac{1}{\tau_{i}}\left(\left(M_{f}\right)_{i, j}-f_{i, j}\right),
$$


where $f_{i, j}=\frac{1}{\left|\Omega_{i}\right|} \int_{\Omega_{i}} f\left(x, \boldsymbol{\xi}_{j}, t\right) d x$ and $\left(M_{f}\right)_{i, j}=\frac{1}{\left|\Omega_{i}\right|} \int_{\Omega_{i}} M_{f}\left(x, \boldsymbol{\xi}_{j}, t\right) d x$, while tau is the relaxation time evaluated at the center of the cell $\Omega_{i}$ : note that this is enough up to second order accuracy. Here, $\sigma$ is the integration variable representing a surface element.

Since a uniform Cartesian grid is considered, the equation in 1D can be simply rewritten in terms of fluxes at each numerical interface (between two cells):

$$
\frac{\partial f_{i, j}}{\partial t}(t)+\frac{1}{\Delta x}\left(F_{i+\frac{1}{2}, j}-F_{i-\frac{1}{2}, j}\right)=\frac{1}{\tau_{i}}\left(\left(M_{f}\right)_{i, j}-f_{i, j}\right),
$$

where $F_{i+\frac{1}{2}, j}$ is the numerical flux between the cell $\Omega_{i}$ and the cell $\Omega_{i+1}$, at the velocity grid node $\xi_{j}$ and can be defined with an upwind discretization as

$$
F_{i+\frac{1}{2}, j}=\max \left(0, \xi_{j}\right) f_{i+1 / 2, j}^{l}+\min \left(0, \xi_{j}\right) f_{i+1 / 2, j}^{r},
$$

with $f^{r}$ and $f^{l}$ the values of $f$ on the two sides of the interface and $\xi_{j}$ the first component of the microscopic velocity. The numerical expression of the distribution functions $f^{l}, f^{r}$ depends on the reconstruction used at the numerical interface. For a first order reconstruction, $f_{i+1 / 2}^{l}=f_{i}$ and $f_{i+1 / 2}^{r}=f_{i+1}$. For second order accuracy, a MUSCL reconstruction with slope limiters (MinMod for example) is employed. Dropping the velocity index $j$, we have

$$
\left\{\begin{array}{l}
f_{i+1 / 2}^{l}=f_{i}+\frac{1}{2} \operatorname{MinMod}\left(f_{i+1}-f_{i}, f_{i}-f_{i-1}\right) \\
f_{i+1 / 2}^{r}=f_{i+1}-\frac{1}{2} \operatorname{MinMod}\left(f_{i+1}-f_{i}, f_{i+2}-f_{i+1}\right)
\end{array}\right.
$$

The second equation for the transport and the relaxation of the rotational temperature is solved with a finite different scheme with upwind fluxes:

$$
\partial_{t} \Theta_{i}=\frac{T_{e q_{i}}-\Theta_{i}}{Z_{r} \tau}-\frac{1}{\Delta x}\left(\frac{U_{i}+\left|U_{i}\right|}{2}\left(\Theta_{i}-\Theta_{i-1}\right)+\frac{U_{i}-\left|U_{i}\right|}{2}\left(\Theta_{i+1}-\Theta_{i}\right)\right) .
$$

Again, this formula can be easily extended to second order accuracy.

\subsection{Time discretization}

The time discretization is performed with an IMEX scheme [5] for the kinetic model and the macroscopic equation. The convective part of the equation is integrated explicitly and the relaxation part implicitly. In this way, the constraint on the time step only comes from the convective part of the equation.

In the following, we drop the indices $i, j$ denoting the space and velocity location of the variables. For simplicity, we focus on the $D$ dimensional reduced model defined in (27), with the Maxwellians defined in (28). The time integration for a $\nu$-stages IMEX Runge-Kutta scheme applied to each kinetic equation composing this model is

$$
\begin{aligned}
& f_{L}^{n+1}=f_{L}^{n}-\Delta t \sum_{k=1}^{\nu} \tilde{\omega}_{k} \boldsymbol{\xi} \nabla_{\mathbf{x}} f_{L}^{(k)}+\frac{\Delta t}{\tau} \sum_{k=1}^{\nu} \omega_{k}\left(M_{f_{L}}^{(k)}-f_{L}^{(k)}\right) \\
& f_{L}^{(k)}=f_{L}^{n}-\Delta t \sum_{l=1}^{k-1} \tilde{A}_{k, l} \boldsymbol{\xi} \nabla_{\mathbf{x}} f_{L}^{(l)}+\frac{\Delta t}{\tau} \sum_{l=1}^{k} A_{k, l}\left(M_{f_{L}}^{(l)}-f_{L}^{(l)}\right),
\end{aligned}
$$

where $L=1,2$ denotes one of the two distribution functions, $A$ and $\tilde{A}$ are $\nu \times \nu$ matrices, with $\tilde{A}_{i, s}=0$ if $s \geq i$ and $A_{i, s}=0$ if $s>i$. These coefficients are derived from the double Butcher's 
tableaux:

$$
\begin{array}{l|l|c}
\tilde{A} \\
\hline \tilde{\omega}^{T}
\end{array} \quad \frac{A}{\omega^{T}}
$$

in which all coefficients composing the IMEX scheme satisfy the correct coupling conditions, guaranteeing the correct accuracy, see $[20,5]$ All the quantities until stage $k-1$ are known so the equation for stage $k$ becomes:

$$
\begin{aligned}
f_{L}^{(k)}= & \frac{\tau}{A_{k, k} \Delta t+\tau}\left(f_{L}^{n}-\Delta t \sum_{l=1}^{k-1} \tilde{A}_{k, l} \boldsymbol{\xi} \nabla_{\mathbf{x}} f_{L}^{(l)}+\frac{\Delta t}{\tau} \sum_{l=1}^{k-1} A_{k, l}\left(M_{f_{L}}^{(l)}-f_{L}^{(l)}\right)\right)+ \\
& \frac{A_{k, k} \Delta t}{\tau+A_{k, k} \Delta t} M_{f_{L}}^{(k)}
\end{aligned}
$$

where $f_{L}^{(k)}, L=1,2$ can be computed immediately, provided one knows the Maxwellian $M_{f_{L}}^{(k)}$ at the stage $k$. In fact, all other quantities involved, which have been gathered in the first parenthesis, are known from previous stages. In the case of a classical BGK model the Maxwellian at the stage $k, M_{f}^{(k)}$ can be computed using the macroscopic variables at the previous stages, see [21]. Here, the evaluation of the Maxwellian is again slightly more complicated, because the different temperatures are not completely defined from the kinetic model.

Computing discrete moments of the second equation in (39), one finds

$$
\left(\begin{array}{c}
\rho^{(k)} \\
\rho^{(k)} \mathbf{U}^{(k)} \\
E^{(k)}
\end{array}\right)=\left(\begin{array}{c}
\rho^{n} \\
\rho^{n} \mathbf{U}^{n} \\
E^{n}
\end{array}\right)-\Delta t \sum_{l=1}^{k-1} \tilde{A}_{k, l}\left\langle\left(\begin{array}{c}
\boldsymbol{\xi} \\
\boldsymbol{\xi} \otimes \boldsymbol{\xi} \\
\frac{1}{2} \boldsymbol{\xi} \boldsymbol{\xi}^{2}
\end{array}\right) \nabla_{\mathbf{x}} f_{1}^{(l)}+\left(\begin{array}{c}
0 \\
0 \\
\boldsymbol{\xi}
\end{array}\right) \nabla_{\mathbf{x}} f_{2}^{(l)}\right\rangle
$$

because the conservation of mass, momentum and total energy, enforced at the discrete level, implies that the moments of the relaxation term is exactly zero. Thus, mass, momentum and the equilibrium temperature $T_{e q}$ can be computed at the time level $(k)$ from quantities known from the previous stages. Note, in particular, that for a first order scheme, with a single time level, we would have that macroscopic quantities remain constant during the relaxation step.

The IMEX step is applied also to (8):

$$
\Theta^{(k)}=\Theta^{n}-\Delta t \sum_{l=1}^{k-1} \tilde{A}_{k, l} \mathbf{U} \nabla_{\mathbf{x}} \Theta^{(l)}+\frac{\Delta t}{Z_{r} \tau} \sum_{l=1}^{k} A_{k, l}\left(T_{e q}{ }^{(l)}-\Theta^{(l)}\right) .
$$

Since $T_{e q}^{(k)}$ is already known, one can easily compute $\Theta^{(k)}, \Lambda^{(k)}$ and complete the construction of $M_{f_{1}}^{(k)}$ and $M_{f_{2}}^{(k)}$. Substituting this information in (40), one obtains $f_{L}^{(k)}, L=1,2$ solving a linear algebraic equation. Note that it is crucial that conservation holds exactly at the discrete level, to ensure that the macroscopic variables do not depend on relaxation. Once all the stages have been computed, the value of $f_{L}^{n+1}, L=1,2$ can be found from the first equation in (39), while $\Theta^{n+1}$ is given by

$$
\Theta^{n+1}=\Theta^{n}-\Delta t \sum_{k=1}^{\nu} \tilde{\omega}_{k} \mathbf{U} \nabla_{\mathbf{x}} \Theta^{(k)}+\frac{\Delta t}{\tau} \sum_{k=1}^{\nu} \omega_{k}\left(T_{e q}{ }^{(k)}-\Theta^{(k)}\right)
$$


Asymptotic Preserving-theorem. The IMEX scheme defined in (39), (42), (43) is Asymptotic Preserving in the sense of [16], namely, as $\tau \rightarrow 0$, it becomes an explicit discretization of the limiting Euler equations.

Proof. We extend the proof appearing in [22]. As $\tau \rightarrow 0$, the system is projected on equilibrium, namely from eq. (39) $f_{L}^{(k)} \rightarrow M_{f_{L}}^{(k)}$, and from eq. (42) $\Theta^{(k)} \rightarrow T_{e q}^{(k)}$. Thus both Maxwellians $M_{f_{L}}$ depend on the same temperature $T_{e q}$. Then the equation for the moments (41) is closed by the Maxwellians, namely,

$$
\left(\begin{array}{c}
\rho^{(k)} \\
\rho^{(k)} \mathbf{U}^{(k)} \\
E^{(k)}
\end{array}\right)=\left(\begin{array}{c}
\rho^{n} \\
\rho^{n} \mathbf{U}^{n} \\
E^{n}
\end{array}\right)-\Delta t \sum_{l=1}^{k-1} \tilde{A}_{k, l} \nabla_{\mathbf{x}}\left\langle\left(\begin{array}{c}
\boldsymbol{\xi} \\
\boldsymbol{\xi} \otimes \boldsymbol{\xi} \\
\frac{1}{2} \boldsymbol{\xi} \xi^{2}
\end{array}\right) M_{f_{1}}^{(l)}+\left(\begin{array}{c}
0 \\
0 \\
\boldsymbol{\xi}
\end{array}\right) M_{f_{2}}^{(l)}\right\rangle
$$

The quadratures in the right hand side can be calculated explicitly. In particular, since all temperatures coincide in the limit $\tau \rightarrow 0$, the energy equation becomes

$$
\left\langle\frac{1}{2} \boldsymbol{\xi} \boldsymbol{\xi}^{2} M_{f_{1}}^{(l)}+\boldsymbol{\xi} M_{f_{2}}^{(l)}\right\rangle=\frac{1}{2} \rho^{(l)}\left(\mathbf{U}^{(l)}\right)^{2}+p^{(l)} \mathbf{U}^{(l)}+\frac{1}{2}(n+m) \rho^{(l)} \mathbf{U}^{(l)} T_{e q}^{(l)},
$$

where we recall that $n$ and $m$ are the number of translational and rotational degrees of freedom, respectively. Since $n+m=d$, we see that we obtain a discretization of the energy flux in the Euler equation, for which the flux is $\frac{1}{2} \rho \mathbf{U}^{2}+(p+\rho e) \mathbf{U}$, with $e$ internal energy per unit mass. Moreover, we also recover the correct $\gamma$-law equation of state. In fact, since at equilibrium all degrees of freedom are endowed with the same energy, because the Maxwellian is isotropic, for each component $i, i=1, n$,

$$
p=<\left[(\boldsymbol{\xi}-\mathbf{U})_{i}\right]^{2} M_{f_{1}}>=\rho T_{e q}
$$

but also $\rho e=\frac{d}{2} \rho T_{e q}$, thus $p=\rho e(\gamma-1)$, with $\gamma=1+2 / d$. The first two equations in (41) give respectively the conservation of mass and momentum. Thus, the equation for the stages of the macroscopic moments become

$$
\left(\begin{array}{c}
\rho \\
\rho \mathbf{U} \\
E
\end{array}\right)^{(k)}=\left(\begin{array}{c}
\rho^{n} \\
\rho^{n} \mathbf{U}^{n} \\
E^{n}
\end{array}\right)-\Delta t \sum_{l=1}^{k-1} \tilde{A}_{k, l} \nabla_{\mathbf{x}}\left(\begin{array}{c}
\rho \\
\rho \mathbf{U} \otimes \mathbf{U}+p \\
\frac{1}{2} \rho \mathbf{U}^{2}+\mathbf{U}\left(p+\frac{d}{2} \rho T_{e q}\right)
\end{array}\right)^{(l)}
$$

Analogously, computing moments of the first equation in (39) for $L=1$, adding the first moment of the equation for $f_{2}$, in the limit $\tau \rightarrow 0$, we obtain

$$
\left(\begin{array}{c}
\rho \\
\rho \mathbf{U} \\
E
\end{array}\right)^{n+1}=\left(\begin{array}{c}
\rho \\
\rho \mathbf{U} \\
E
\end{array}\right)^{n}-\Delta t \sum_{k=1}^{\nu} \tilde{\omega}_{k} \nabla_{\mathbf{x}}\left(\begin{array}{c}
\rho \\
\rho \mathbf{U} \otimes \mathbf{U}+p \\
\frac{1}{2} \rho \mathbf{U}^{2}+\mathbf{U}\left(p+\frac{d}{2} \rho T_{e q}\right)
\end{array}\right)^{(k)} .
$$

The last two equations are an explicit Runge Kutta discretization of the Euler equations, with a $\nu$ stages explicit RK scheme, defined by the Butcher tableau given by the coefficients $\tilde{\omega}_{k}$ and $\tilde{A}_{k, l}$.

\section{Numerical results}

Most numerical test cases are simulations of the flow of nitrogen $\left(N_{2}\right)$, which is a bi-atomic gas with a viscosity coefficient $\omega=0.72$. In this section, P-BGK will denote the polyatomic BGK model proposed in this work. First and second order numerical schemes in space are used with a first order in time (for computational time reasons). 


\subsection{Sod shock tube}

We start illustrating the effect of the Asymptotic Preserving property of the scheme. We consider a shock tube problem, for a polyatomic gas. Here we will consider the reduced 1D model, with a total of $d$ degrees of freedom, with $m=d-3$. Since $\tau$ is very small, the temperatures are very close, irrespective of $Z_{r}$. Here, the space [0,1] is discretized with 100 grid points. In velocity, the space goes to -10 to 10 and discretized with 50 grid points. $Z_{r}$ is taken from the model 7 and $\tau=10^{-5}$.

$$
\left\{\begin{array} { l } 
{ \rho _ { l } = 1 } \\
{ u _ { l } = 0 } \\
{ p _ { l } = 1 }
\end{array} \quad \text { and } \quad \left\{\begin{array}{l}
\rho_{r}=0.125 \\
u_{r}=0 \\
p_{r}=0.1
\end{array}\right.\right.
$$
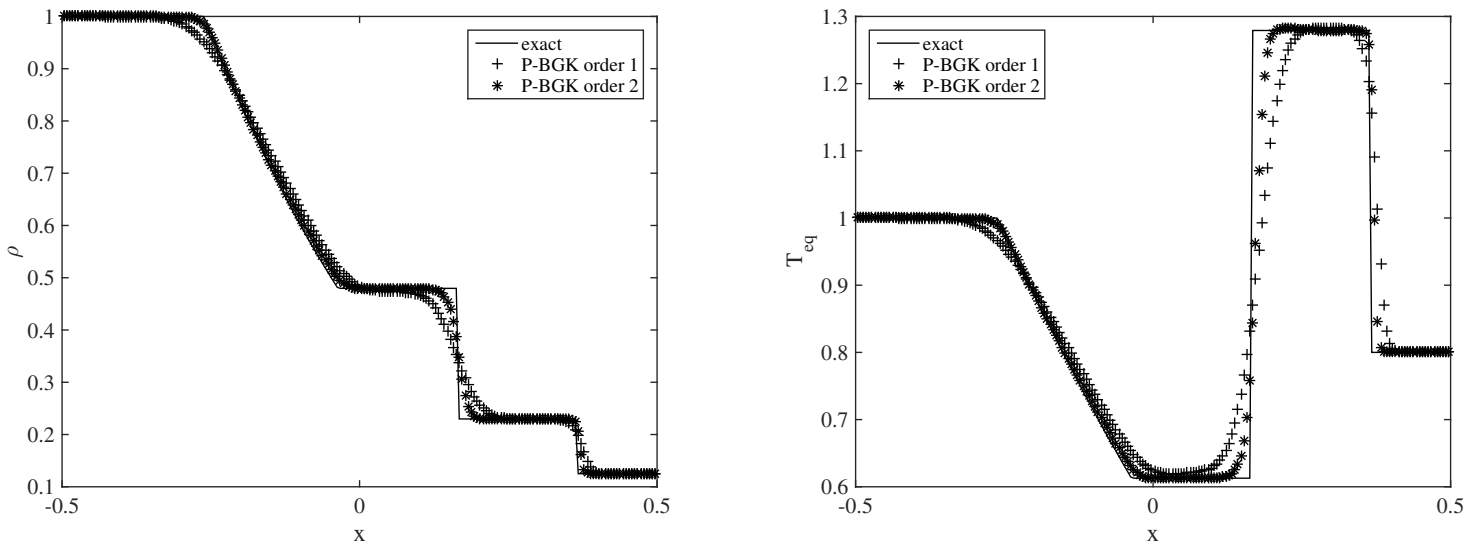

Figure 1: Comparison between exact and P-BGK solutions for $\gamma=5 / 3$. Density (left) and Temperature (right) profiles. First and second order scheme.
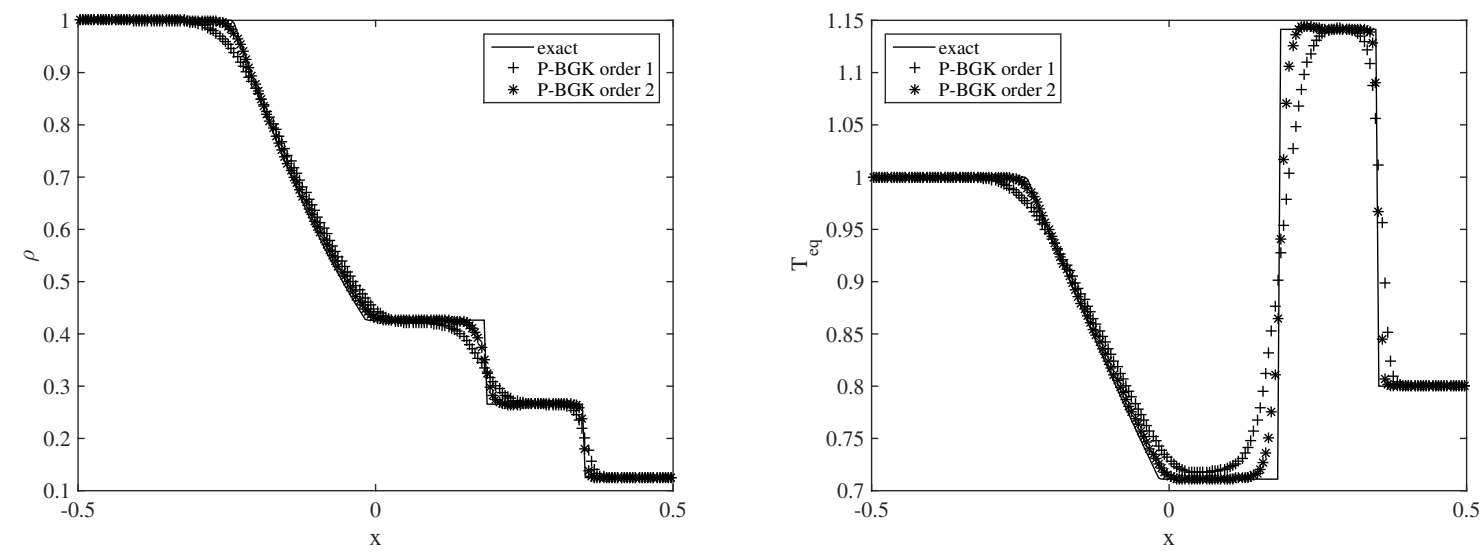

Figure 2: Comparison between exact and P-BGK solutions for $\gamma=7 / 5$. Density (left) and Temperature (right) profiles. First and second order scheme.

We show the results for several values of $m$, comparing with the exact hydrodynamic solution of the Riemann problem, with the corresponding value of $\gamma=1+\frac{2}{d}$. The results appear in Fig. 1 (monoatomic case), Fig. 2 (bi-atomic molecule), but also in Fig. 3 (a polyatomic molecule with 2 rotational degrees of freedom and one vibrational) and Fig. 4 for a polyatomic molecule with four internal degrees of freedom. 

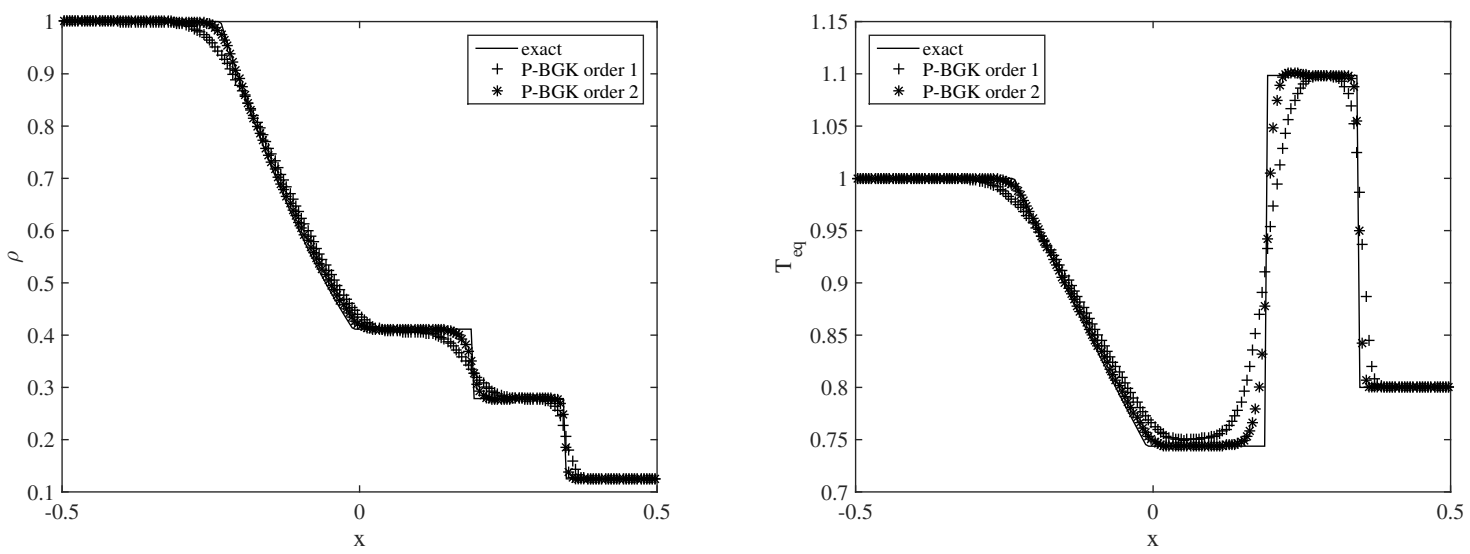

Figure 3: Comparison between exact and P-BGK solutions for $\gamma=4 / 3$. Density (left) and Temperature (right) profiles. First and second order scheme.
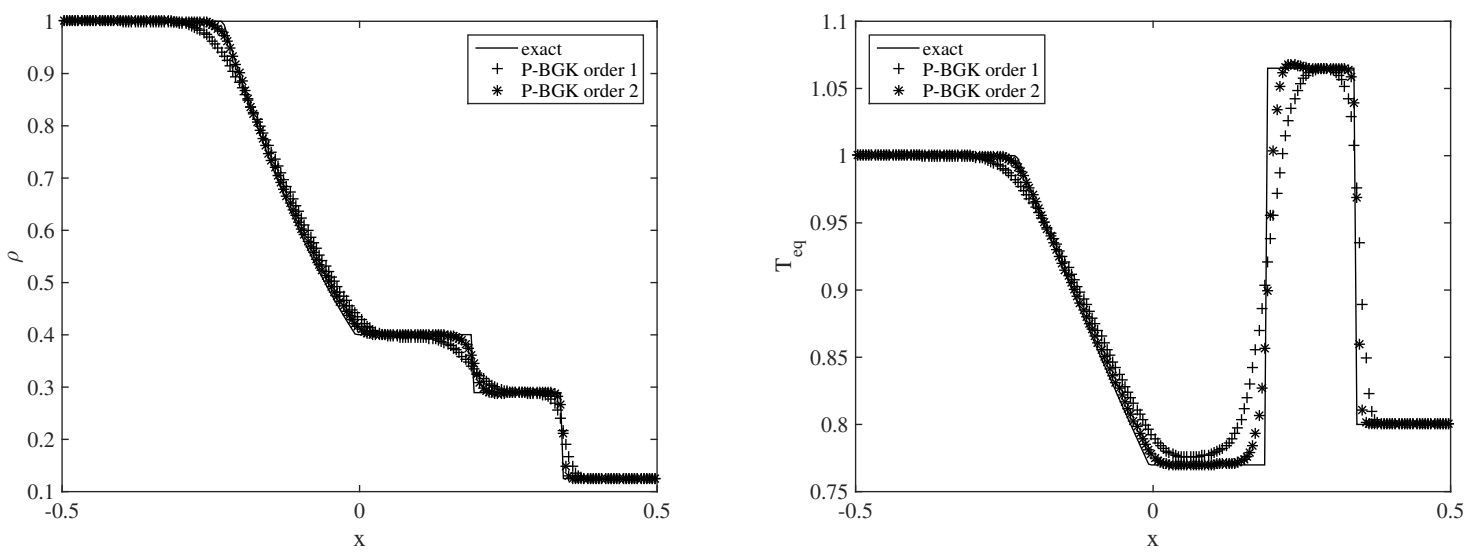

Figure 4: Comparison between exact and P-BGK solutions for $\gamma=9 / 7$. Density (left) and Temperature (right) profiles. First and second order scheme.

It is clear from the figures that the kinetic scheme provides indeed a discretized solution of the Euler equations, with the correct value of $\gamma$. Note also the sharpening of the discontinuities in the solution from the first to the second order scheme. This effect is particularly noticeable on the contact discontinuity.

\subsection{A stationary shock}

The set up of this test consists of initial conditions resulting in a Mach 2.2 stationary shock located in $x=0$. The initial conditions are the following:

$$
\left\{\begin{array} { l } 
{ \rho _ { l } = 1 } \\
{ u _ { l } = C \sqrt { \gamma } } \\
{ p _ { l } = ( \rho _ { l } u _ { l } ^ { 2 } - \rho _ { r } u _ { r } ^ { 2 } ) \frac { \gamma + 1 - \frac { \rho _ { r } } { \rho _ { l } } ( \gamma - 1 ) } { 2 \gamma ( \frac { \rho _ { r } } { \rho _ { l } } - 1 ) } }
\end{array} \quad \text { and } \quad \left\{\begin{array}{l}
\rho_{r}=C \\
u_{r}=\sqrt{\gamma} \\
p_{r}=p_{l} \frac{\frac{\rho_{r}}{\rho_{l}}(\gamma+1)-(\gamma-1)}{\gamma+1-\frac{\rho_{r}}{\rho_{l}}(\gamma-1)}
\end{array}\right.\right.
$$

with $C$ a coefficient depending on the Mach number in the left state expressed as

$$
C=\frac{2+M^{2}(\gamma-1)}{(\gamma+1) M^{2}} .
$$


Here, $M=2.2$ and it corresponds to the Mach number on the left side of the shock.

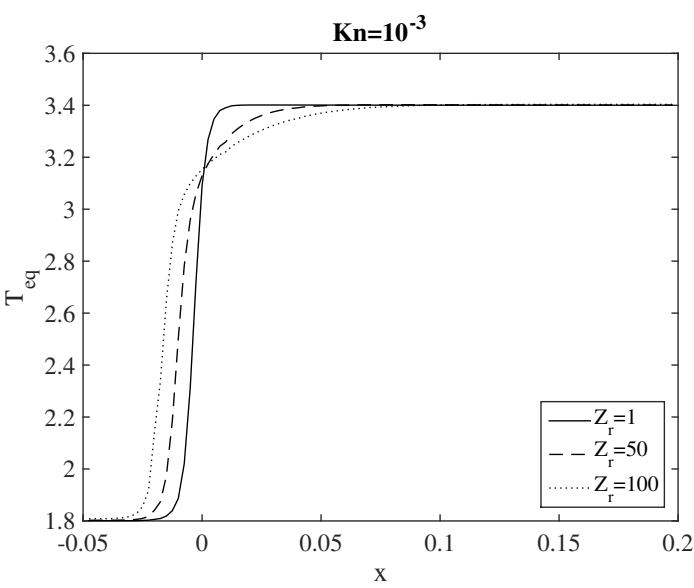

(a) Equilibrium temperature for different values of $Z_{r}(1,50,100)$ and $\mathrm{Kn}=10^{-3}$

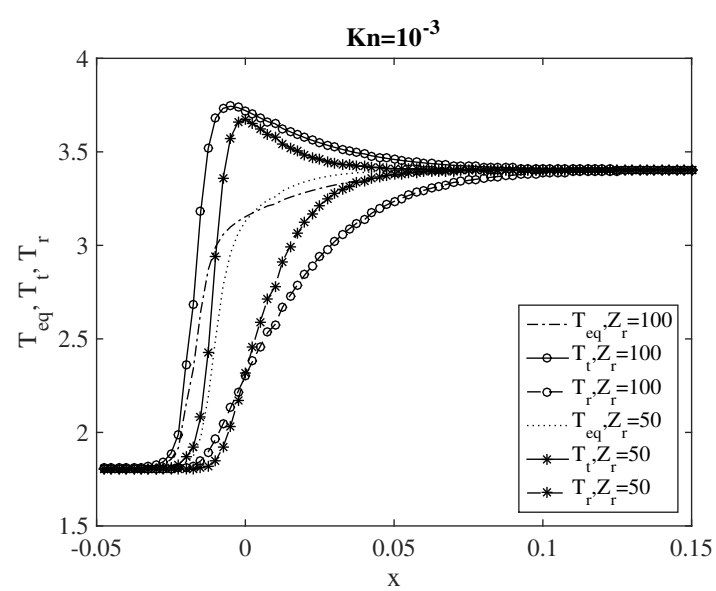

(b) Equilibrium, translational and rotational temperatures for different values of $Z_{r}(50,100)$ and $\mathrm{Kn}=10^{-3}$

Figure 5: Temperature profiles for different values of $Z_{r}$

The relaxation time $\tau$ is chosen as $\tau=\mathrm{Kn}=10^{-3}$, which means that the flow will be in equilibrium away from the shock, but within the shock, non equilibrium effects will still be noticeable.

Figure 5a shows the equilibrium temperature for $\mathrm{Kn}=10^{-3}$ and different values of $Z_{r}$. One can see that the shock structure is modified as $Z_{r}$ increases, slowing down the convergence to equilibrium. In particular, the shock becomes more diffused and is not symmetric with respect to $x=0$. Figure $5 \mathrm{~b}$ shows the different temperature profiles for $Z_{r}=50,100$. Around the shock, the energy is more concentrated in the translational degrees of freedom, which decay fater. As $Z_{r}$ increases, the translational temperature increases and it is greater than the equilibrium temperature, as also observed in [18]. After the shock the translational and the rotational temperature both relaxe towards the equilibrium temperature, because the flow is close to equilibrium.

This test case emphasizes that non-equilibrium phenomena, can lead to a wrong estimation of the heat fluxes after a shock, when only the equilibrium temperature is taken into account. In the case of a bi-atomic gas, the rotational temperature can be 2.5 times larger than the equilibrium temperature (if the translational temperature is 0 then $T_{e q}=2 / 5 * T r$ ) and the translational temperature 1.6 times larger (if the rotational temperature is 0 then $T_{e q}=5 / 3 * T_{t}$ ), modifying drastically the heat flux.

\subsection{Comparison with experimental results}

We consider again a shock tube problem, resulting in a stationary shock. We consider $M=2$ and $M=10$ in eq. (48) and (49), in order to compare our results with [1] where experimental and DSMC results are shown for stationary shocks with these Mach numbers. $T t_{l}, T t_{r}, T r_{l}, T r_{r}$ are the initial translational and rotational temperature on the left and on the right of the shock. In practice, $T t_{l}=T r_{l}$ and $T t_{r}=T r_{r}$. Here, to fit with the experimental set up of Alsmeyer and simulate the same conditions, we take $K n=0.662$. It is not clear how the parameter $Z r$ is chosen so we just took it from the empirical expression 7 .

The results obtained with the P-BGK model are in good accordance with the experimental and the DSMC results for the density profile. Both profiles fit well with the DSMC results. 


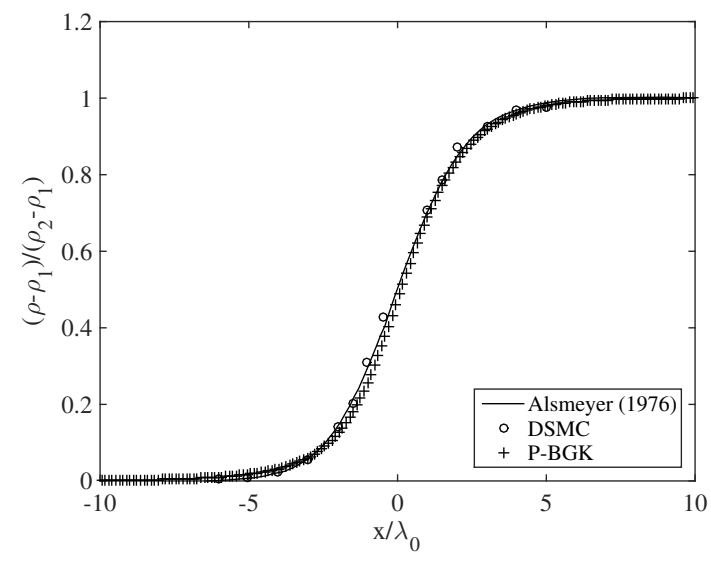

(a) $\mathrm{M}=2$

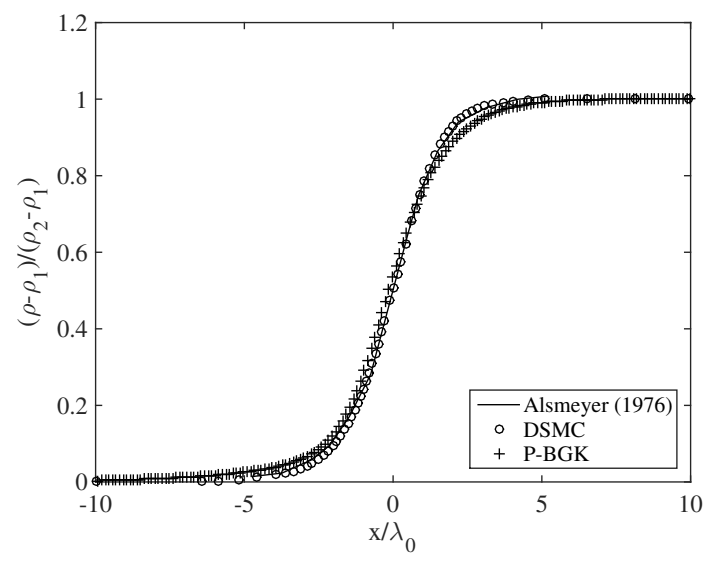

(b) $\mathrm{M}=10$

Figure 6: Density profiles obtained with DSMC, experiments and the P-BGK model.

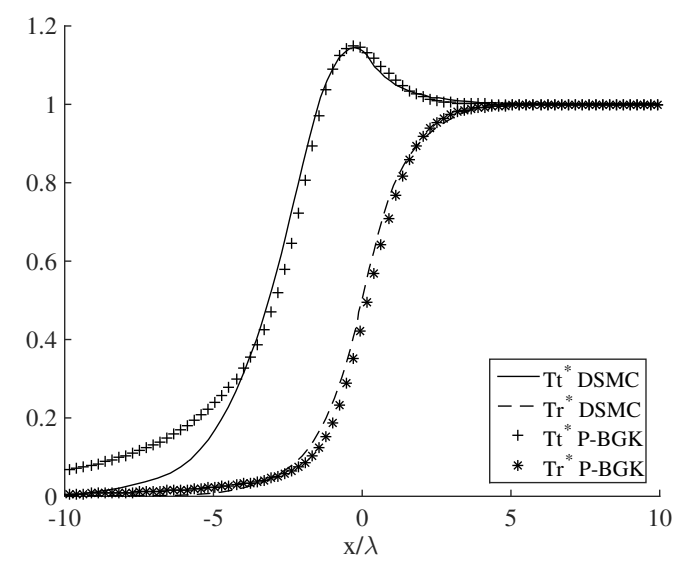

Figure 7: Translational and rotational normalized temperatures. $T t^{*}=\left(T t-T t_{l}\right) /\left(T t_{r}-T t_{l}\right)$ and $T r^{*}=\left(T r-T r_{l}\right) /\left(T r_{r}-T r_{l}\right)$

Discrepancies can be observed in the temperature profiles, Fig. 7. It is apparent that the translational temperature anticipates the shock for our model. It also affects the equilibrium temperature since the energy is conserved and the rotational temperature does not compensate the early increasing. However, the general behaviour and the peak are well captured. The difference can be due to the uncertainty on the relaxation time for the rotational temperature, and also to the need for a more refined approach, such as an ES-BGK extension of the present model. The temperature profiles for $\mathrm{M}=2$ are not shown here since there are no data in [1] for this Mach number.

\subsection{Ringleb flow}

Now, we consider a 2D test for a bi-atomic gas close to the continuum regime, where we can compare our results with $2 \mathrm{D}$ Euler solutions. We take $\tau$ close to zero $\left(10^{-5}\right)$, so that the kinetic gas is close to equilibrium with $Z_{r}$ form 7 .

In this test, we consider Ringleb flow, which is a $2 \mathrm{D}$ steady solution, where the analytical solution can be calculated for Euler equations and will be used as a reference solution for the kinetic model close to the hydrodynamic regime. 


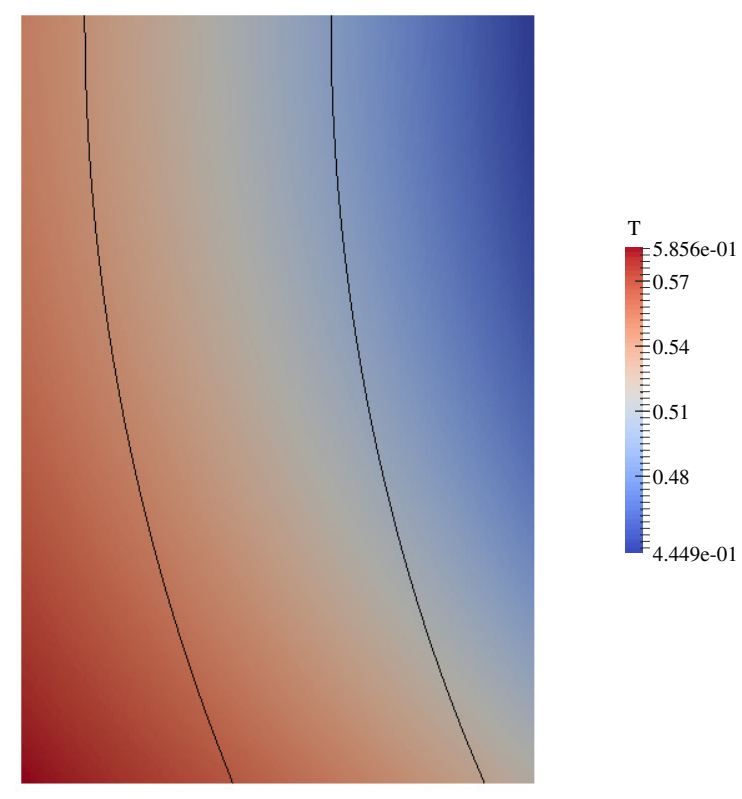

Figure 8: Equilibrium temperature field for Ringleb flow.

The Euler flow is potential, and the exact solution is obtained with the hodograph method, [25]. Setting $(\theta, \mathrm{V})$ such that $u=V \cos \theta$ and $v=V \sin \theta$, the stream function is given by $\Psi=\frac{\sin \theta}{V}$. The streamline equations are:

$$
x=\frac{1}{2 \rho}\left(\frac{1}{V^{2}}-2 \Psi^{2}\right)+\frac{L}{2} \quad \text { and } \quad y=\frac{\sin \theta \cos \theta}{\rho V^{2}}
$$

with (for $\gamma=7 / 5$, diatomic gas):

$$
L=\ln \left(\frac{3 V}{6+2 \sqrt{9-3 V^{2}}}\right)-\frac{\sqrt{9-3 V^{2}}\left(V^{2}-4\right)}{V^{2}-3}, \quad c^{2}=1-\frac{\gamma-1}{2} V^{2}, \quad \rho=c^{\frac{2}{\gamma-1}} .
$$

The computational domain is $[-0.5,-0.1] \times[-0.6,0]$ and the flow is solved between the two streamlines $\Psi_{1}=0.8$ and $\Psi_{2}=0.9$. Since $\mathbf{U} \cdot \mathbf{n}=0$ on a streamline (with $\mathbf{n}$ the normal to the streamline), any streamline can be considered as a solid boundary where the Euler impermeability condition is enforced. The boundary conditions in inlet $(\mathrm{y}=-0.6)$ and outlet $(\mathrm{y}=0)$ are supersonic and exactly imposed. The Knudsen number is set to $10^{-5}$, and we take $\tau=\mathrm{Kn}$. The physical space is discretized with $256 \times 384$ points. The velocity space goes from -14 to 14 and discretized with 21 grid points in each direction. First order schemes are used.

Fig. 8 shows the temperature field in the steady state Ringleb solution for the kinetic solution. We also show the two streamlines delimiting the computational domain. Note that there are no spurious effects at the boundary. The boundary conditions in fact are imposed with the AP boundary condition from [8].

The convergence study towards the exact equilibrium solution is found in Fig. 9a and 9b for the $L^{1}$ and the $L^{\infty}$ norm of the error respectively. Convergence is studied under grid refinement for the first order scheme, on a few macroscopic variables, namely, the two components of the velocity, pressure and the sound speed. 


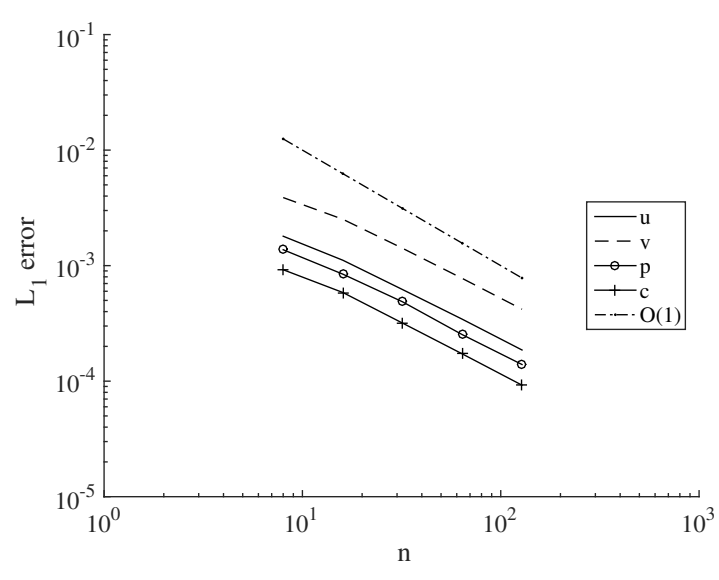

(a) $L_{1}$ norm.

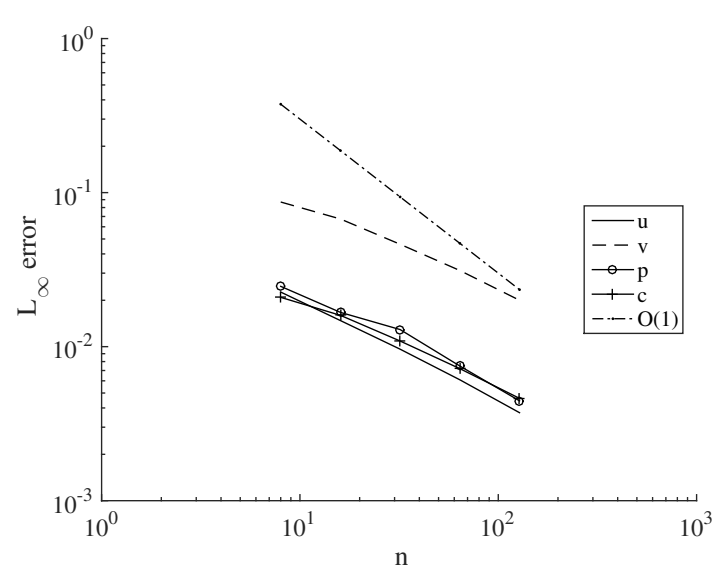

(b) $L_{\infty}$ norm.

Figure 9: Convergence towards the analytical Euler solution in $L_{1}$ and $L^{\infty}$ norm.

\subsection{Blunt body at $\mathrm{M}=5$}

We consider a Blunt body in a flow at $M=5$ in $2 \mathrm{D}$. The Blunt body has a radius of 0.01 units, and the domain is $[-0.05,0] \times[0,0.05]$ discretized with 100 cells in each direction. The reduced velocity field is $[-14,14] \times[-14,14]$ discretized with 33 cells in each direction. On the Blunt body a diffuse boundary condition is imposed with $T=1$ and $\mathbf{U}=0$ considering the gas close to the wall at equilibrium $\left(\Theta_{w}=\Lambda_{w}=1\right)$. The Knudsen number in reference condition is $\mathrm{Kn}=10^{-3}$, while $\tau$ is given in (2) and the exponent of the viscosity law is set to $\delta=0.72$ as for nitrogen. The coefficient $Z_{r}$ is calculated from the formula given in [18].

Figure 10 shows the solution for the equilibrium temperature.

The temperature profiles obtained with the polyatomic BGK model are plotted along the stagnation line and compared with results from the literature. In particular, we superpose our data with the DSMC and UGKS (Unified Gas Kinetic Scheme) from [18] for a bi-atomic molecule. Finally, the equilibrium temperature can be found in Fig. 12. Again, note that the results are indeed very close.

\section{Conclusion}

In this work, we have proposed a simple kinetic model for polyatomic molecules, based on the BGK approach, in which it is possible to account for different relaxation rates along the internal energy degrees of freedom of a single molecule.

The model can be reduced, grouping together similar degrees of freedom, extending the technique of [15]. It introduces additional distribution functions to treat internal degrees of freedom. However, the size of the phase space remains constant with respect to the monoatomic BGK model. Moreover, we also extend the numerical scheme of [21], constructing a scheme that has a stability restriction of CFL type, given only by the convective terms of the equation, but not by the relaxation time. This permits to obtain an efficient numerical method, which still is able to account for the different energy relaxation times.

We prove an $\mathrm{H}$-theorem for this model, thus guaranteeing the convergence towards a unique equilibrium state, determined by the initial conditions, for an isolated gas. We also prove that the IMEX scheme proposed is Asymptotic Preserving, and thus converges to a discretization of 


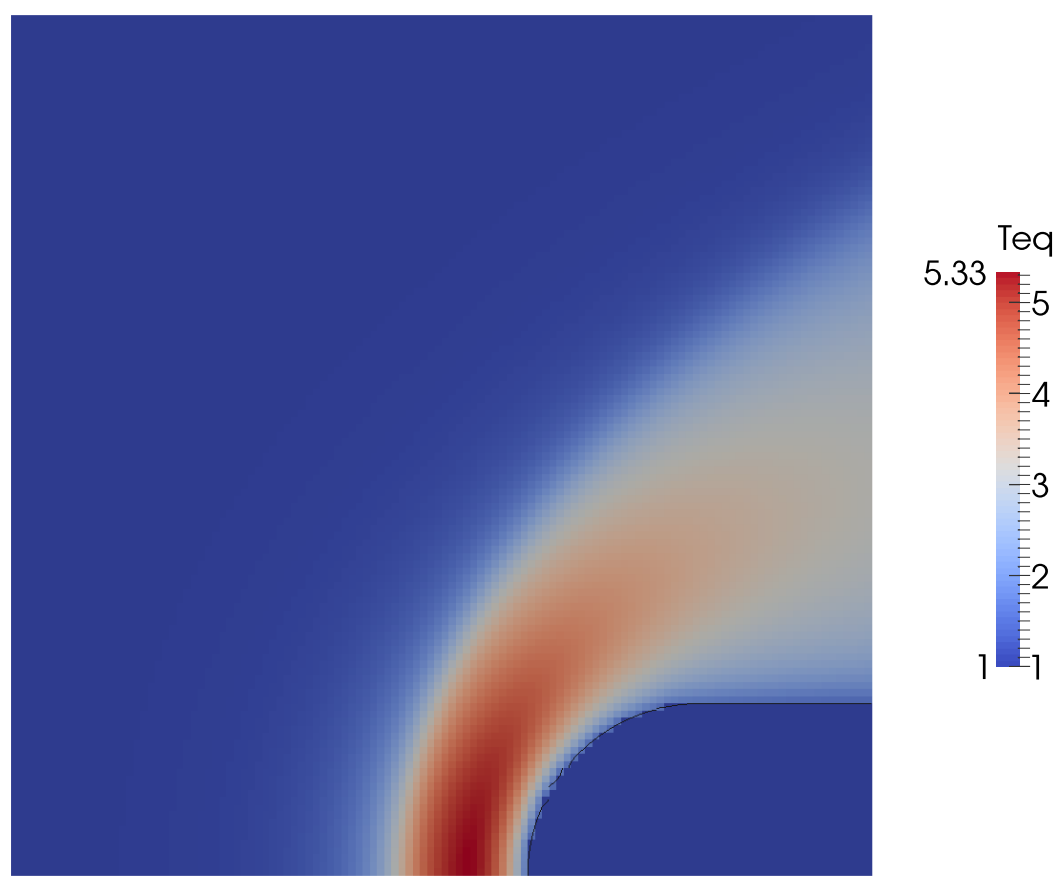

Figure 10: Equilibrium temperature

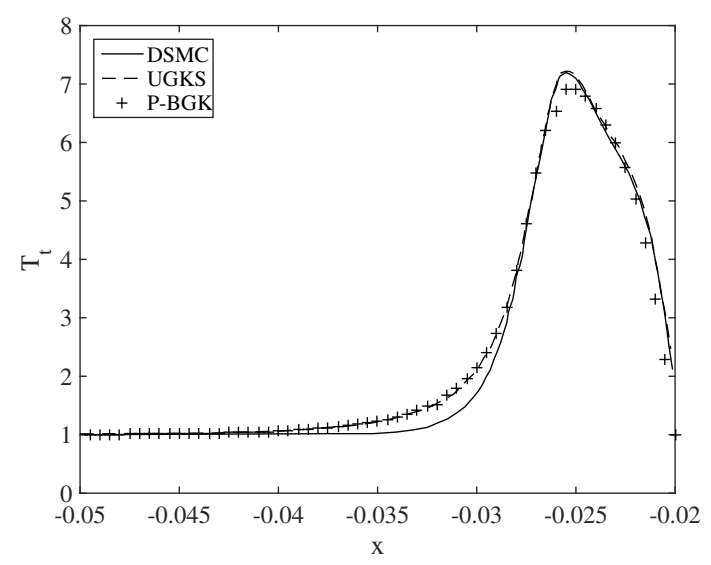

(a) Translational temperature

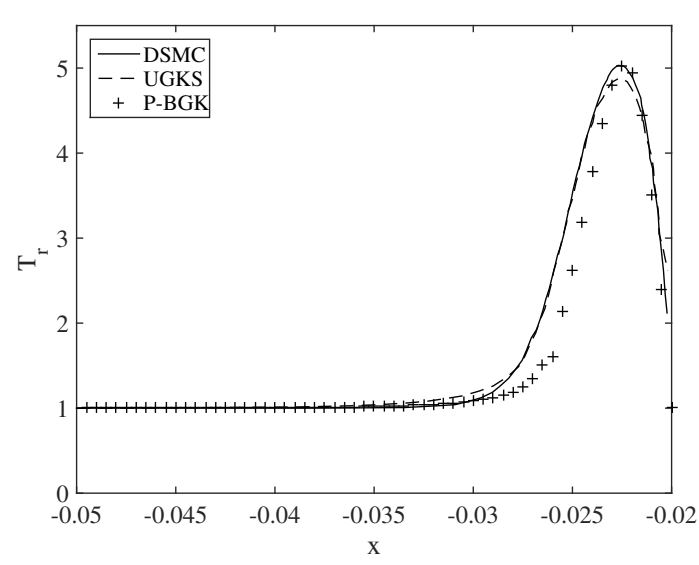

(b) Rotational temperature

Figure 11: Temperature profiles for the Blunt body problem. Comparison between DSMC, UGKS, and the P-BGK results.

the correct hydrodynamic equations, as the relaxation time goes to zero.

The study is documented with several one and two-dimensional tests, which illustrate the properties of the model and of the numerical scheme. We reproduce Riemann problems for several values of the polytropic constant $\gamma$, we study the shock structure of a stationary shock, and also more classical two dimensional problems from gas dynamics.

Future extensions of this work will concentrate on a ES-BGK extension of the present PBGK polyatomic model. The purpose is to represent more effectively, at moderate Knudsen numbers, the viscous and thermal exchanges within the gas. 


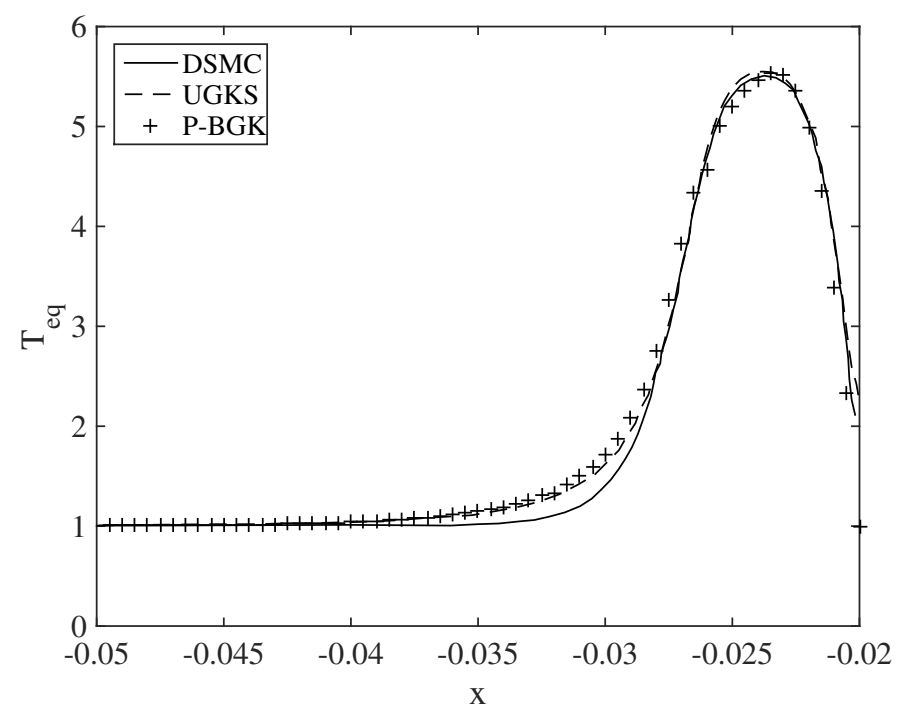

Figure 12: Equilibrium temperature for the Blunt body problem. Comparison between DSMC, UGKS, and the P-BGK results.

\section{Acknowledgements}

Experiments presented in this paper were carried out using the PlaFRIM experimental testbed, being developed under the Inria PlaFRIM development action with support from LABRI and IMB and other entities: Conseil Régional d'Aquitaine, FeDER, Université de Bordeaux and CNRS (see https://plafrim.bordeaux.inria.fr/).

This study has been carried out with financial support from the French State, managed by the French National Research Agency (ANR) in the frame of the "Investments for the future" Programme IdEx Bordeaux (ANR-10-IDEX-03-02), Cluster of excellence CPU, and partly by the Italian State under GNCS-INDAM 2015 project "Metodi numerici per la quantificazione dell'incertezza in equazioni iperboliche e cinetiche".

\section{References}

[1] Alsmeyer, H. Density profiles in argon and nitrogen shock waves measured by the absorption of an electron beam. Journal of Fluid Mechanics (1976). (Cited on pages 17 and 18.)

[2] Andries, P., Bourgat, J.-F., Le Tallec, P., and Perthame, B. Numerical comparison between the Boltzmann and ES-BGK models for rarefied gases. Computer Methods in Applied Mechanics and Engineering 191, 31 (May 2002), 3369-3390. (Cited on page 2.)

[3] Andries, P., Le Tallec, P., Perlat, J.-P., and Perthame, B. The Gaussian-BGK model of Boltzmann equation with small Prandtl number. European Journal of Mechanics. B. Fluids 19, 6 (2000), 813-830. (Cited on pages 3, 4, and 7.)

[4] Andries, P., and Perthame, B. The ES-BGK model equation with correct Prandtl number. 22nd International Symposium on Rarefied Gas Dynamics. AIP Conference Proceeding 585 (2001), 30. (Cited on page 2.) 
[5] Ascher, U. M., Ruuth, S. J., And Spiteri, R. J. Implicit-explicit Runge-Kutta methods for time-dependent partial differential equations. Applied Numerical Mathematics. An IMACS Journal 25, 2-3 (1997), 151-167. (Cited on pages 12 and 13.)

[6] Bernard, F. Efficient Asymptotic Preserving Schemes for BGK and ES-BGK models on Cartesian grids. Theses, Politecnico di Torino, Université de Bordeaux, Mar. 2015. (Cited on page 11.)

[7] Bernard, F., Iollo, A., And Puppo, G. A Local Velocity Grid Approach for BGK Equation. Communications in Computational Physics 16, 4 (2014), 956-982. (Cited on page 11.)

[8] Bernard, F., Iollo, A., And Puppo, G. Accurate Asymptotic Preserving Boundary Conditions for Kinetic Equations on Cartesian Grids. Journal of Scientific Computing 65 (Nov. 2015), 735-766. (Cited on pages 11 and 19.)

[9] Bhatnagar, P. L., Gross, E. P., And Krook, M. A Model for Collision Processes in Gases. I. Small Amplitude Processes in Charged and Neutral One-Component Systems. Phys. Rev. 94 (May 1954), 511-525. (Cited on page 1.)

[10] Borgnakke, C., and Larsen, P. S. Statistical collision model for Monte Carlo simulation of polyatomic gas mixture. Journal of Computational Physics 18 (Aug. 1975), 405-420. (Cited on page 2.)

[11] Brull, S., And Schneider, J. On the ellipsoidal statistical model for polyatomic gases. Continuum Mechanics and Thermodynamics 20, 8 (Mar. 2009), 489-508. (Cited on page 2.)

[12] Cabannes, H., Gatignol, R., and Luol, L.-S. The discrete Boltzmann equation. Lecture Notes at University of California, Berkley (Jan. 1980), 1-65. (Cited on page 11.)

[13] Cercignani, C. The Boltzmann Equation and Its Applications. Springer-Verlag GmbH, 1988. (Cited on page 1.)

[14] Cercignani, C., Illner, R., And Pulvirenti, M. The Mathematical Theory of Dilute Gases. No. v. 106 in Applied Mathematical Sciences Series. Springer-Verlag GmbH, 1994. (Cited on page 2.)

[15] Chu, C. K. Kinetic-theoretic description of the formation of a shock wave. Phys. Fluids 8 (1965), 12-22. (Cited on pages 2, 8, and 20.)

[16] Jin, S. Asymptotic preserving (AP) schemes for multiscale kinetic and hyperbolic equations: a review. Rivista di Matematica della Università di Parma. Serie 7 3, 2 (2012), 177-216. (Cited on pages 2 and 14.)

[17] Liu, C., Xu, K., Sun, Q., And CaI, Q. A unified gas-kinetic scheme for continuum and rarefied flows IV: Full Boltzmann and model equations. Journal of Computational Physics 314 (Mar. 2016), 305-340. (Cited on page 2.)

[18] Liu, S., Yu, P., Xu, K., And Zhong, C. Unified gas-kinetic scheme for diatomic molecular simulations in all flow regimes. Journal of Computational Physics 259, C (Feb. 2014), 96-113. (Cited on pages 4, 5, 17, and 20.)

[19] Mieussens, L. Discrete-Velocity Models and Numerical Schemes for the Boltzmann-BGK Equation in Plane and Axisymmetric Geometries. Journal of Computational Physics 162, 2 (Aug. 2000), 429-466. (Cited on page 11.) 
[20] Pareschi, L., And Russo, G. Implicit-Explicit Runge-Kutta Schemes and Applications to Hyperbolic Systems with Relaxation. Journal of Scientific Computing 25, 1 (Oct. 2005), 129-155. (Cited on page 13.)

[21] Pieraccini, S., And Puppo, G. Implicit-Explicit schemes for BGK kinetic equations. Journal of Scientific Computing 32, 1 (2007), 1-28. (Cited on pages 2, 13, and 20.)

[22] Pieraccini, S., ANd Puppo, G. Microscopically implicit-macroscopically explicit schemes for the BGK equation. Journal of Computational Physics (2011). (Cited on page 14.)

[23] Rykov, V. A model kinetic equation for a gas with rotational degrees of freedom. Fluid Dyn. 6, 10 (1975), 956-966. (Cited on page 2.)

[24] Semyonov, Y., Borisov, P., And Suetin, P. Investigation of heat transfer in rarefied gases over a wide range of Knudsen number. Int. J. Heat Mass Transfer 10 (Mar. 1984), 1789-1799. (Cited on page 2.)

[25] Shapiro, A. The Dynamics and Thermodynamics of Compressible Fluid Flow. Ronald Press, 1953. (Cited on page 19.)

[26] Tantos, C., Frezzotti, A., Valougeorgis, D., and Morini, G. L. Conductive heat transfer in a rarefied polyatomic gas confined between coaxial cylinders. International Journal of Heat and Mass Transfer 79 (1997), 378-389. (Cited on page 2.)

[27] Xu, K., Xin, H., And Chunpei, C. Multiple temperature kinetic model and gas-kinetic method for hypersonic non equilibrium flow computations. Journal of Computational Physics 227 (Apr. 2008), 6779-6794. (Cited on page 2.) 